\title{
Model solution for volume reflection of relativistic particles in a bent crystal
}

\author{
M.V. Bondarenco* \\ Kharkov Institute of Physics and Technology, 1 Academic St., 61108 Kharkov, Ukraine
}

(Dated: March 21, 2022)

\begin{abstract}
For volume reflection process in a bent crystal, exact analytic expressions for positively- and negatively-charged particle trajectories are obtained within a model of parabolic continuous potential in each interplanar interval, with the neglect of incoherent multiple scattering. In the limit of the crystal bending radius greatly exceeding the critical value, asymptotic formulas are obtained for the particle mean de?ection angle in units of Lindhards critical angle, and for the final beam profile. Volume re?ection of negatively charged particles is shown to contain effects of rainbow scattering and orbiting, whereas with positively charged particles none of these effects arise within the given model. The model predictions are compared with experimental results and numerical simulations. Estimates of the volume re?ection mean angle and the final beam profile robustness under multiple scattering are performed.
\end{abstract}

PACS numbers: $61.85 .+\mathrm{p}, 29.27 .-\mathrm{a}, 45.10 .-\mathrm{b}$

Keywords: volume reflection; harmonic inter-planar potential; rainbow scattering; orbiting

\section{INTRODUCTION}

The volume re?ection is an effect of deflection of highenergy charged particles upon their over-barrier (nonchanneled) passage through a planarly oriented bent crystal. The effect arises when the crystal bending radius $R$ greatly exceeds the critical value $R_{c}$. That condition is the same as the Tsyganovs one for the possibility of channeling in a bent crystal [1], but the particle motion regime in the crystal yet depends on the particle entry angle relative to the active atomic planes. When this angle is much larger than the critical value $\theta_{c}$, then, moving in the continuous potential of bent planes, conserving the particle full transverse (radial) energy, the particles are rarely captured into channels (via incoherent scattering on atomic electrons and nuclei), and are mostly deflected elastically through the volume reflection mechanism. $\mathrm{Cu}-$ riously, the latter deflection proceeds to the side opposite to that of the crystal bending; the value of the deflection angle is of the order of critical angle $\theta_{c}$. Furthermore, the particle beam after deflection remains fairly well collimated, i.e., its angular dispersion keeps much smaller than the mean deflection angle. That phenomenon was discovered in numerical simulations two decades ago 2] and recently verified experimentally [3, 4]. Nowadays it is considered to be an option for beam collimation and partial extraction at ultrarelativistic charged-particle accelerators [5] 7 .

To a good accuracy, the particle dynamics in the volume reflection problem is classical 8 and reduces to classical particle motion in a cylindrically symmetrical continuous potential of bent atomic planes. Therewith, granted the angular momentum conservation relative to the active crystallographic plane bending axis, the final deflection angle is expressible in the standard way as an

${ }^{*}$ Electronic address: bon@kipt.kharkov.ua integral over the radial coordinate from an inverse square root function involving the potential [see Eq. (55b) below]. That approximation served as a starting point for a number of numerical studies [2, 9].

Although the described computational problem seems to be sufficiently simple, it is aggravated by the presence of several parameters: the ratio $R / R_{c}$, initial and final particle variables (impact parameter, the angles of incidence and deflection). The dependencies on all those parameters involve singularities, which in general are better dealt with by analytic techniques than by numerical ones. Besides that, as long as for practice mostly interesting is the case $R \gg R_{c}$, it would be instructive to evaluate the asymptotic behavior of all relevant observables in the formal limit $R / R_{c} \rightarrow \infty$, including next-to-leading order corrections in the small parameter $R_{c} / R$. But since volume reflection depends on the particle dynamics not in one but in several inter-planar intervals, for feasibility of its global analytic description one rather needs some model.

A valuable opportunity for realistic model building is that the inter-planar potential in a silicon crystal, at least in the orientation (110), is fairly close to parabolic shape over the entire inter-planar interval (see, e. g., [10]). A parabolic (harmonic) potential, i.e., a linear oscillator, permits a simple solution for the particle trajectory within a single interplanar interval. The next problem is to connect solutions on the boundaries of the adjacent intervals. It may appear nontrivial, but it is feasible to do that transitively, i.e., simultaneously for an arbitrary number of the adjacent intervals. Thereby we obtain a completely solvable model capturing basic features of the volume re?ection, except the effects of incoherent multiple scattering.Moreover, we are able to derive not only the de?ection angle, but also an expression for the whole trajectory, which further on may be used for description of inelastic processes, such as volume capture or electromagnetic radiation.

In the present work we will deliver a solution for the 
posed model problem. The plan of the article is as follows. In Sec. [II we describe the procedure of solution connection between adjacent inter-planar intervals, demonstrating that the problem reduces to elementary trigonometry. The particle trajectory is expressed as an explicit function of inter-planar interval order number (not involving a recursive procedure), for arbitrary ratio $R / R_{c}$. In Sec. III from the obtained solution for trajectory, we derive the particle final deflection angle, which comes as a sum of inverse trigonometric (for positively charged particles) or hyperbolic (for negatively charged particles) functions. In Sec. IV] we scrutinize the limit $R \gg R_{c}$, most interesting in relation to volume reflection and practical applications, first for positively, then for negatively charged particles. In the generic expression for the ?nal deflection angle, we find a possibility to replace the sums involved by integrals (via the EulerMaclaurin formula), and do the latter ones in a closed form. As a result, we arrive at sufficiently simple asymptotic formulas for the de?ection angle dependence on all the variables. The impact parameters are thereupon analytically averaged over, and the experimentally observable scattering differential cross section is obtained for positive and negative particles. In Sec. $\nabla$ we examine the opposite limit $R \ll R_{c}$. In Sec. [V] we provide estimates of optimal crystal and initial beam parameters for beam complete deflection or for experimental investigation of the final beam profile features. A summary is given in Sec. VII.

\section{PARTICLE TRAJECTORY IN A BENT CRYSTAL}

\section{A. Initial conditions}

The usual geometry of experiments on volume reflection implies sending a charged particle beam normally to a thin [20], weakly bent crystal plate. The practically unavoidable slight curvature of the crystal boundary thereat is of minor consequence, since the main contribution to the particle reflection angle comes from a vicinity of some point in the depth of the crystal. For definiteness and to establish an easy connection with the particle impact parameter in the initial (perfectly parallel [21]) beam, let us consider a particle incident along the $z$ axis on a crystal whose front face is a perfect plane, located at $z=0$. As for the crystal rear face, for our purposes in this paper we may leave it unspecified at all, as if the crystal was infinitely thick, but transparent. Then, let $\theta_{0}\left(0<\theta_{0} \ll 1\right)$ be the angle of inclination of crystalline planes to the $z$ axis at the crystal front face (see Fig. 1), and let $x$-axis, perpendicular to $O z$, point in the direction of the crystal bend. Moving at small angles to the crystal planes, the particle interacts most strongly (coherently) with the averaged, so-called continuous inter-planar potential [8], which induces a force with dominant $x$-component (yet slowly dependent on $z$ ) [22]. Along the $y$ coordinate there

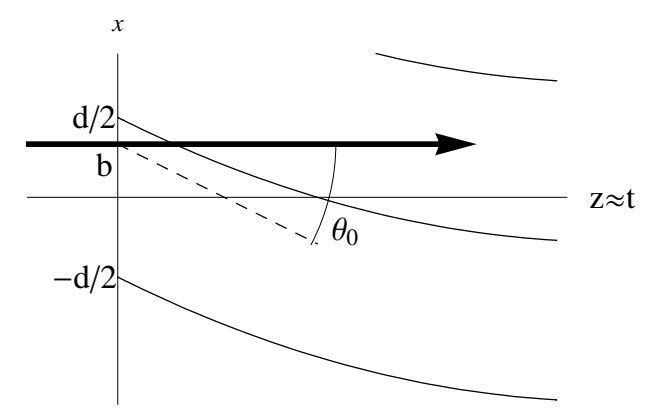

FIG. 1: Coordinates describing the bent crystal geometry (circle segments are the bent atomic planes) and the particle entrance to the crystal (thick arrow). Not to scale. For details see text.

is a translational invariance, ensuring the particle momentum $y$-component conservation.

In monocrystals of not too heavy chemical elements, in particular for silicon (lattice of diamond-type), oriented by its (110) plane close to the direction of the beam, the continuous potential in each inter-planar interval may closely be approximated by a quadratic function, with an accuracy $\lesssim 20 \%[23]$. That entails a linear equation of motion for the classical 24] ultra-relativistic [25] particle:

$$
\begin{gathered}
\ddot{x}=\frac{2 F_{\max }}{E d}\left(-x+x_{0}\right), \\
t \approx z \quad(c=1, \text { small angle motion }),
\end{gathered}
$$

where $x_{0}$ is the midpoint of the inter-planar interval, $d$ the inter-planar distance, $E$ the particle energy, and $F_{\max }$ the force acting on the particle at the edge of the interplanar interval $x-x_{0}=-\frac{d}{2}$. For positively charged particles $F_{\max }$ is positive, whereas for negatively charged particles it is negative. Note that the force and the particle energy enter equation (1) only through the ratio

$$
\frac{E}{\left|F_{\max }\right|}=R_{c}
$$

known as the Tsyganov critical radius [1]. The natural time unit in channeling-related phenomena is

$$
\tau=\sqrt{\frac{E d}{2\left|F_{\max }\right|}} \equiv \sqrt{\frac{R_{c} d}{2}}
$$

( $2 \pi \tau$ has the meaning of positively charged particle channeling period, although herein we deal not with channeling but with an over-barrier motion).

One and the only consequence of the crystal bending is that $x_{0}$ in Eq. (11) acquires dependence on the longitudinal coordinate $z$, which for ultra-relativistic motion under small angles to $O z$ may be equated to the current time $t$ :

$$
x_{0}=x_{0}(z \approx t) \quad \text { (the crystal bend function). }
$$


In application to volume reflection, we are interested in the uniform bending of the crystal, at which $x_{0}(t)$ describes a circular arc of a small opening angle. That small arc may equally well be approximated by a parabola, and hence $x_{0}(t)$ is determined by the equation

$$
x_{0}(t)=-\theta_{0} t+\frac{t^{2}}{2 R} \quad \text { (uniformly bent crystal), }
$$

where $R$ is the atomic plane bending radius (without the loss of generality one may let $x_{0}(0)=0-$ see Fig. 1). Inserting (6) to (11), and implementing (4), we get the particle equation of motion in the first inter-planar interval:

$\ddot{x}= \pm \frac{1}{\tau^{2}}\left(-x-\theta_{0} t+\frac{t^{2}}{2 R}\right) \quad\left\{\begin{array}{l}\text { pos. charged particles } \\ \text { neg. charged particles }\end{array}\right\}$.

Initial conditions for $x(t)$ stand as

$$
\begin{aligned}
& x(0)=b, \\
& \dot{x}(0)=0,
\end{aligned}
$$

where $b$, restricted to the interval

$$
-\frac{d}{2} \leq b \leq \frac{d}{2}
$$

is the impact parameter measured from the middle of the interval

The equations of motion further simplify in terms of the "subtracted radius" variable

$$
r(t)=-x(t)-\theta_{0} t+\frac{t^{2}}{2 R},
$$

becoming

$$
\ddot{r}=\frac{\delta \mp r}{\tau^{2}} \quad\left(\text { in }-\frac{d}{2} \leq r \leq \frac{d}{2}\right),
$$

where

$$
\delta=\frac{\tau^{2}}{R}
$$

Thus, $\pm \delta$ is the spatial shift of the oscillator equilibrium position due to the crystal bend, i. e., due to the centrifugal force, which in the present small-angle approximation, presuming condition $r \ll R$ within the weakly bent crystal, is treated as virtually independent of the subtracted radius $r$ (cf. [2]). For $r(t)$, the initial conditions (8) 9) translate to

$$
\begin{aligned}
& r(0)=-b, \\
& \dot{r}(0)=-\theta_{0} .
\end{aligned}
$$

Generic solution of Eq. (12) reads:

$$
r_{0}(t)= \pm \delta-A_{0}\left\{\begin{array}{c}
\sin \\
\sinh
\end{array}\right\}\left(\frac{t}{\tau}+\varphi_{0}\right) .
$$

(Here and henceforth upper signs and figures refer to positively charged particles, and lower ones - to negatively charged particles.) Matching initial conditions (14 15) allows one to determine constants $A_{0}$ and $\varphi_{0}$ :

$$
\begin{aligned}
A_{0} & =\sqrt{\tau^{2} \theta_{0}^{2} \pm(b \pm \delta)^{2}}, \\
\varphi_{0} & =\left\{\begin{array}{l}
\arcsin \\
\operatorname{arsinh}
\end{array}\right\} \frac{b \pm \delta}{A_{0}}
\end{aligned}
$$

(the sign of $A_{0}$ must be chosen positive in order that $\dot{r}(0)$ at $\theta_{0}>0$, according to (15), was negative).

Further on, solution (16) is to be connected with solutions in the subsequent inter-planar intervals. Importantly, since these solutions have to be connected at a definite $r$, the condition of connection will not depend on the current phase of the harmonic motion, such as $\varphi_{0}$ in Eq. (16), as we are going to show.

\section{B. Connection of solutions through interval borders}

Moving along trajectory (16), the particle will cross the next inter-planar interval border $r=-\frac{d}{2}$ at an instant

$$
\frac{t_{1}}{\tau}=\left\{\begin{array}{l}
\arcsin \\
\operatorname{arsinh}
\end{array}\right\} \frac{\frac{d}{2} \pm \delta}{A_{0}}-\varphi_{0}
$$

(inferred from (16) by letting $r=-\frac{d}{2}$ and solving for $t$ ). At this instant, the equation of particle motion turns to

$$
\ddot{r}=\frac{\delta \mp(d+r)}{\tau^{2}} \quad\left(\text { in }-\frac{3 d}{2} \leq r \leq-\frac{d}{2}\right) .
$$

That is the same harmonic oscillator, only with an altered equilibrium position, and general solution of (20) may be written as

$$
r_{1}(t)= \pm \delta-d-A_{1}\left\{\begin{array}{c}
\sin \\
\sinh
\end{array}\right\}\left(\frac{t}{\tau}+\varphi_{0}+\Delta \varphi_{1}\right) .
$$

Values of new constants $A_{1}, \triangle \varphi_{1}$ are now to be determined from the continuity of $r(t)$ and $\dot{r}(t)$ at the border point $r=-\frac{d}{2}$. That can be done without formally solving the system of two equations. First, compare two integrals of motion

$$
\begin{aligned}
& A_{1}^{2}=\tau^{2} \dot{r}_{1}^{2} \pm\left( \pm \delta-d-r_{1}\right)^{2} \\
& A_{0}^{2}=\tau^{2} \dot{r}_{0}^{2} \pm\left( \pm \delta-r_{0}\right)^{2}
\end{aligned}
$$

(related to transverse energy) in their common point, where $r_{0}=r_{1}=-\frac{d}{2}, \dot{r}_{0}=\dot{r}_{1}$. Subtracting (23) from (22), one gets $A_{1}^{2}=A_{0}^{2}-2 \delta d$, i. e.

$$
A_{1}=\sqrt{A_{0}^{2}-2 \delta d}
$$




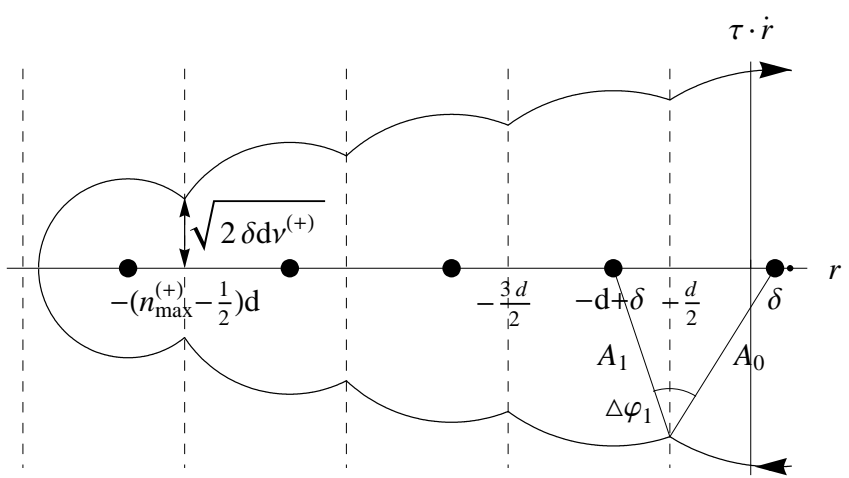

FIG. 2: Solid curve - the phase-space (the subtracted radius $r$ vs. the radial velocity $\dot{r}$ ) trajectory for positively charged particles, under condition $\delta<\frac{d}{2}$. Dashed vertical lines signify the positions of bent atomic planes (definite $r$ ). Thick dots indicate centers of the trajectory circular segments. Vertex angle $\Delta \varphi_{1}$ (and similarly all other $\Delta \varphi_{n}$ ) may be interpreted as a geometric sum of vertex angles in a pair of right triangles having a common cathetus, and with the second catheti equal $\frac{d}{2}+\delta, \frac{d}{2}-\delta$, and the hypotenuses $A_{0}, A_{1}$.

Thereupon, the phase shift $\Delta \varphi_{1}$ is sought from a condition $r_{1}\left(t_{1}\right)=-\frac{d}{2}$. One finds:

$$
\Delta \varphi_{1}=-\left\{\begin{array}{c}
\arcsin \\
\operatorname{arsinh}
\end{array}\right\} \frac{\frac{d}{2} \pm \delta}{A_{0}}-\left\{\begin{array}{l}
\arcsin \\
\operatorname{arsinh}
\end{array}\right\} \frac{\frac{d}{2} \mp \delta}{A_{1}}
$$

(for a geometric interpretation of this relation for positive particles - see Fig. 2). As we had expected, neither $A_{1}$, nor $\Delta \varphi_{1}$ depends on $\varphi_{0}$.

At each next border connection of the solutions is implemented in exactly the same way. Writing in the $n$-th interval

$r_{n}(t)= \pm \delta-n d-A_{n}\left\{\begin{array}{c}\sin \\ \sinh \end{array}\right\}\left(\frac{t}{\tau}+\varphi_{0}+\sum_{m=1}^{n} \Delta \varphi_{m}\right)$,

$$
\left(-\frac{d}{2}-n d \leq r \leq \frac{d}{2}-n d, \quad t_{n} \leq t \leq t_{n+1}\right)
$$

the generic amplitude is found as

$$
\begin{aligned}
A_{n} & =\sqrt{A_{n-1}^{2}-2 \delta d}=\sqrt{A_{0}^{2}-2 n \delta d} \\
& =\sqrt{\tau^{2} \theta_{0}^{2} \pm(b \pm \delta)^{2}-2 n \delta d} .
\end{aligned}
$$

and the phase shift is deduced to be

$$
\triangle \varphi_{n}=-\left\{\begin{array}{l}
\arcsin \\
\operatorname{arsinh}
\end{array}\right\} \frac{\frac{d}{2} \pm \delta}{A_{n-1}}-\left\{\begin{array}{l}
\arcsin \\
\operatorname{arsinh}
\end{array}\right\} \frac{\frac{d}{2} \mp \delta}{A_{n}}
$$

where the amplitudes in the denominators must be treated as already known, by (27). The instants of border

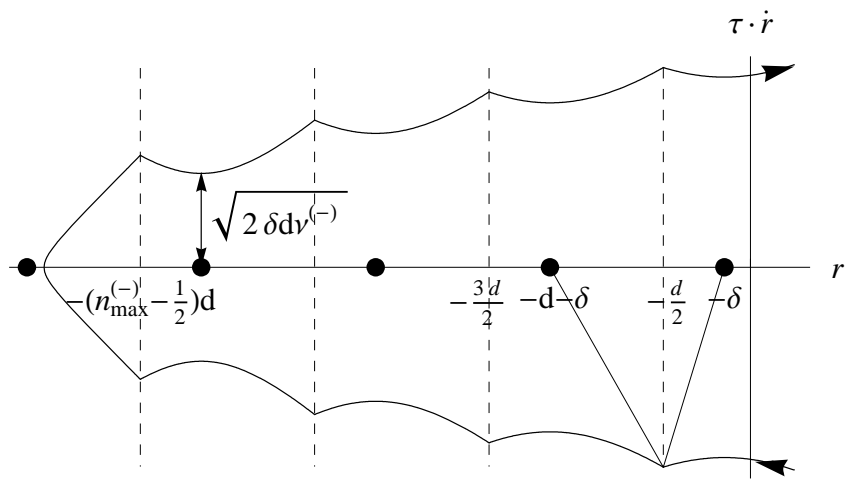

FIG. 3: The same as Fig. 2 but for negatively charged particles, assuming condition $\delta<\frac{d}{2}$ (see Sec. IIIB).

passage can also be evaluated:

$$
\begin{aligned}
\frac{t_{n}}{\tau}= & \sum_{m=1}^{n}\left\{\begin{array}{l}
\arcsin \\
\operatorname{arsinh}
\end{array}\right\} \frac{\frac{d}{2} \pm \delta}{A_{m-1}}+\sum_{m=1}^{n-1}\left\{\begin{array}{l}
\arcsin \\
\operatorname{arsinh}
\end{array}\right\} \frac{\frac{d}{2} \mp \delta}{A_{m}} \\
& -\left\{\begin{array}{l}
\arcsin \\
\operatorname{arsinh}
\end{array}\right\} \frac{b \pm \delta}{A_{0}} . \quad\left(r_{n-1} \rightarrow r_{n}\right)
\end{aligned}
$$

One caution is that amplitudes $A_{n}$ should not be regarded as a measure of the particle spatial wiggling in each interval. As Figs. 2, 3indicate, the trajectory swinging enhances as the particle penetrates deeper into the crystal, whereas amplitudes $A_{n}$, to the contrary, decrease. There is no contradiction hereat because for most of the intervals traversed the intra-channel oscillation period $2 \pi \tau$ is much greater than the time of particle passage across the interval, thus the particle is far from making a full oscillation in each interval, anyway. In fact, the lower is the amplitude $A_{n}$ compared to the interval length (along the particle motion direction), the stronger warp of the trajectory on this interval may occur (see Figs. 2 , 3).

\section{PARTICLE REFLECTION}

\section{A. Reflection conditions for positive particles}

It is clear that the decrease of amplitudes (27) can not continue indefinitely, because eventually arguments of the arcsines in (28) shall exceed unity (that happens sooner than the radicand in (27) becomes negative). This merely signals that the particle can not reach the next inter-planar interval. The particle will continue the harmonic motion until it hits the previous interval, then proceeds moving outwards in the radial variable in the same but reverse way, and on the exit from the crystal it will emerge as a deflected beam.

Let us evaluate the order number $n_{\max }^{(+)}$of the reflection interval. If for some $n$ inequality $\frac{d}{2}+\delta \leq A_{n-1}$ is met, then it also entails $\frac{d}{2}-\delta \leq A_{n}$, so arguments of all 
the arcsines in (29) are less than unity. So, $n_{\max }^{(+)}$is the largest integer yet allowing for $\frac{d}{2}-\delta \leq A_{n_{\max }^{(+)}}$. Through (27), that condition determines the reflection interval order number:

$$
n_{\max }^{(+)}=\left\lfloor\frac{\tau^{2} \theta_{0}^{2}+(b+\delta)^{2}-\left(\frac{d}{2}-\delta\right)^{2}}{2 \delta d}\right\rfloor,
$$

where the lower-corner brackets $\lfloor\ldots\rfloor$ designate the integer part of a number $(\lfloor a\rfloor \leq a)$. If $\tau \theta_{0} \gg d$, variation of $n_{\max }(b)$ is much smaller than its mean value

$$
n_{\max } \sim \frac{\tau^{2} \theta_{0}^{2}}{2 \delta d}=\frac{R}{2 d} \theta_{0}^{2}
$$

(essentially valid for negative particles as well - see Eq. (51) below). It is instructive to notice that

$$
\frac{d}{2}-\delta \leq A_{n_{\max }^{(+)}}<\frac{d}{2}+\delta .
$$

Toward the volume reflection problem, we are interested in finding the total reflection angle $\theta_{\text {refl }}$, half of which, by symmetry reasons, amounts to deflection angle of the trajectory in the reflection point $t=t_{\text {refl }}$ in which

$$
\dot{r}\left(t_{\text {refl }}\right)=0,
$$

i. e.,

$$
\frac{1}{2} \theta_{\mathrm{refl}} \simeq \dot{x}\left(t_{\mathrm{refl}}\right)=-\theta_{0}+\delta \frac{t_{\mathrm{refl}}}{\tau^{2}}
$$

(more exactly - see Eq. (52) below) 26]. To evaluate the right-hand side of (33), one only needs to know the value of $t_{\text {reff }}$. The latter is found from solving equation $\dot{r}\left(t_{\text {refl }}\right)=0$ :

$$
\begin{aligned}
\frac{t_{\mathrm{refl}}}{\tau}= & \frac{t_{n_{\max }^{(+)}}}{\tau}+\frac{\pi}{2} \\
= & \frac{\pi}{2}+\sum_{n=1}^{n_{\max }^{(+)}}\left(\arcsin \frac{\frac{d}{2}+\delta}{A_{n-1}}+\arcsin \frac{\frac{d}{2}-\delta}{A_{n}}\right) \\
& -\arcsin \frac{b+\delta}{A_{0}} .
\end{aligned}
$$

The largest contribution to the emerging sum comes from the terms $n \sim n_{\max }^{(+)}$(where denominators $A_{n}$ are smallest), so it may be more convenient to revert here the summation order. Introducing a useful parameter

$$
\nu^{(+)}=\left\{\frac{\tau^{2} \theta_{0}^{2}+(b+\delta)^{2}-\left(\frac{d}{2}-\delta\right)^{2}}{2 \delta d}\right\}_{\mathrm{f}},
$$

with braces $\{\ldots\}_{\mathrm{f}}$ to indicate the fractional part $(0 \leq$ $\left.\nu^{(+)}<1\right)$, one recasts (34) as

$$
\begin{aligned}
\frac{t_{\text {refl }}}{\tau}= & \frac{\pi}{2}-\arcsin \frac{b+\delta}{\sqrt{\left(\frac{d}{2}-\delta\right)^{2}+2\left(\nu^{(+)}+n_{\max }^{(+)}\right) \delta d}} \\
& +\sum_{n=0}^{n_{\max }^{(+)}-1}\left(\arcsin \frac{\frac{d}{2}+\delta}{\sqrt{\left(\frac{d}{2}+\delta\right)^{2}+2\left(\nu^{(+)}+n\right) \delta d}}\right. \\
& \left.+\arcsin \frac{\frac{d}{2}-\delta}{\sqrt{\left(\frac{d}{2}-\delta\right)^{2}+2\left(\nu^{(+)}+n\right) \delta d}}\right) \cdot \quad(36 \mathrm{a})
\end{aligned}
$$

Equivalently, using the identity $\arcsin \frac{1}{\sqrt{1+\eta}}=\operatorname{arccot} \sqrt{\eta}$, one can write

$$
\begin{aligned}
& \frac{t_{\text {refl }}}{\tau}=\frac{\pi}{2}-\arcsin \frac{b+\delta}{\sqrt{\left(\frac{d}{2}-\delta\right)^{2}+2\left(\nu^{(+)}+n_{\max }^{(+)}\right) \delta d}} \\
& +\sum_{n=0}^{n_{\text {max }}^{(+)}-1}\left(\operatorname{arccot} \frac{\sqrt{2\left(\nu^{(+)}+n\right) \delta d}}{\frac{d}{2}+\delta}+\operatorname{arccot} \frac{\sqrt{2\left(\nu^{(+)}+n\right) \delta d}}{\frac{d}{2}-\delta}\right) .
\end{aligned}
$$

The physical meaning of parameter $\nu^{(+)}$is clear from Fig. 2. It represents the kinetic transverse energy at the last atomic plane before the reflection, divided by the centrifugal potential difference between the neighboring atomic planes.

\section{B. Negative particles}

In contrast to trigonometric arc-sine, hyperbolic arcsine function exists at any value of its argument. Therefore, expression (26) for negatively charged particle trajectories holds until the radicand in the motion amplitude $A_{n}$ given by (27) becomes negative. The first interval at which that happens will be called "inflection" one. Its order number is inferred to be

$$
n_{\text {infl }}=\left\lfloor\frac{\tau^{2} \theta_{0}^{2}-(b-\delta)^{2}}{2 \delta d}\right\rfloor+1 .
$$

In the inflection interval the amplitude $A_{n_{\text {infl }}}$ calculated by the formula (27) would be imaginary. That implies that the $r(t)$ dependence now is to be described by a hyperbolic cosine rather than a sine (hence the term "inflection"). Matching the amplitude and the phase of the hyperbolic cosine with solution (26) for the preceding $n=n_{\text {infl }}-1$ gives

$$
\begin{array}{r}
r_{n_{\text {infl }}}(t)=-\delta-n_{\text {infl }} d+\mid A_{n_{\text {infl }} \mid} \cosh \left(\frac{t}{\tau}+\varphi_{0}+\sum_{m=1}^{n_{\text {infl }}-1} \triangle \varphi_{m}\right. \\
\left.-\operatorname{arsinh} \frac{\frac{d}{2}-\delta}{A_{n_{\text {infl }}-1}}-\operatorname{arcosh} \frac{\frac{d}{2}+\delta}{\left|A_{n_{\text {infl }} \mid}\right|}\right) \\
\left(-\frac{d}{2}-n_{\text {infl }} d \leq r_{n_{\text {infl }}} \leq \frac{d}{2}-n_{\text {infl }} d\right)
\end{array}
$$


where

$$
\begin{aligned}
\left|A_{n_{\mathrm{infl}}}\right| & =\sqrt{-\tau^{2} \theta_{0}^{2}+(b-\delta)^{2}+2 n_{\mathrm{infl}} \delta d} \\
& \equiv \sqrt{2 \delta d\left(1-\nu^{(-)}\right)}
\end{aligned}
$$

with

$$
\nu^{(-)}=\left\{\frac{\tau^{2} \theta_{0}^{2}-(b-\delta)^{2}}{2 \delta d}\right\}_{\mathrm{f}} .
$$

Since $\frac{d}{2}+\delta \geq \sqrt{2 \delta d}$, the argument of arcosh in (38) is always $\geq 1$.

Next, a question arises, at which condition the trajectory (38) can actually reach the next interval, i. e. $r_{n_{\text {infl }}}(t)$ can descend to value $n_{\text {inff }} d-\frac{d}{2}$. Since by Eq. (40), $r_{n_{\text {infl }}}(t) \geq-\delta-n_{\text {infl }} d+\left|A_{n_{\text {infl }}}\right|$, that would require

$$
\delta-\frac{d}{2}>\left|A_{n_{\mathrm{infl}}}\right| .
$$

Substituting here (39), and solving with respect to the ratio $\frac{\delta}{d}$, one may present (41) in form

$$
\frac{\delta}{d} \equiv \frac{R_{c}}{2 R}>f\left(\nu^{(-)}\right)
$$

with

$$
f\left(\nu^{(-)}\right)=\frac{3}{2}-\nu^{(-)}+\sqrt{\left(2-\nu^{(-)}\right)\left(1-\nu^{(-)}\right)} .
$$

Function $f\left(\nu^{(-)}\right)$decreases monotonously (almost linearly) from $f(0)=\frac{3}{2}+\sqrt{2} \approx 2.9$ to $f(1)=\frac{1}{2}$.

In the simplest case illustrated in Fig. 3, when condition (42) is violated (e. g., if $\frac{\delta}{d}<\frac{1}{2} \leq f$ ), (37) must be the last interval reached by the particle, its order number being

$n_{\max }^{(-)}=n_{\text {infl }}=\left\lfloor\frac{\tau^{2} \theta_{0}^{2}-(b-\delta)^{2}}{2 \delta d}\right\rfloor+1 \quad\left(\frac{\delta}{d} \leq f\left(\nu^{(-)}\right)\right)$.

Expressing $t_{\text {refl }}$ from equation $\dot{r}\left(t_{\text {refl }}\right)=0$ then gives

$$
\begin{aligned}
& \frac{t_{\text {refl }}}{\tau}=\operatorname{arcosh} \frac{\frac{d}{2}+\delta}{\left|A_{n_{\text {infl }}}\right|}-\operatorname{arsinh} \frac{b-\delta}{A_{0}} \\
& +\sum_{n=0}^{n_{\text {infl }}-1} \operatorname{arsinh} \frac{\frac{d}{2}-\delta}{A_{n}}+\sum_{n=1}^{n_{\text {infl }}-1} \operatorname{arsinh} \frac{\frac{d}{2}+\delta}{A_{n}} .
\end{aligned}
$$

Reversal of the summation order here leads to an expression

$$
\begin{aligned}
\frac{t_{\text {refl }}}{\tau}=\operatorname{arcosh} & \frac{\frac{d}{2}+\delta}{\sqrt{2 \delta d\left(1-\nu^{(-)}\right)}}-\operatorname{arsinh} \frac{b-\delta}{A_{0}} \\
& +\sum_{n=0}^{n_{\text {infl }}-1} \operatorname{arsinh} \frac{\frac{d}{2}-\delta}{\sqrt{2 \delta d\left(\nu^{(-)}+n\right)}} \\
& +\sum_{n=0}^{n_{\text {infl }-2}} \operatorname{arsinh} \frac{\frac{d}{2}+\delta}{\sqrt{2 \delta d\left(\nu^{(-)}+n\right)}} .
\end{aligned}
$$

Therethrough, using Eq. (33), results the deflection angle.

Otherwise, i. e. if (42) holds (e. g., if $\frac{\delta}{d}>3>f$ ), in all the subsequent intervals after (37) the trajectory must also express through hyperbolic cosines:

$$
\begin{gathered}
r_{n}(t)=-\delta-n d+\left|A_{n}\right| \cosh \left(\frac{t}{\tau}+\varphi_{0}+\sum_{m=1}^{n_{\text {infl }}-1} \Delta \varphi_{m}\right. \\
-\operatorname{arsinh} \frac{\frac{d}{2}-\delta}{A_{n_{\text {infl }}-1}}-\operatorname{arcosh} \frac{\frac{d}{2}+\delta}{\mid A_{n_{\text {infl }} \mid}} \\
\left.-\sum_{m=n_{\text {infl }}+1}^{n} \operatorname{arcosh} \frac{\frac{d}{2}+\delta}{\left|A_{m}\right|}+\sum_{m=n_{\text {infl }}}^{n-1} \operatorname{arcosh} \frac{-\frac{d}{2}+\delta}{\left|A_{m}\right|}\right) \\
\left(-\frac{d}{2}-n d \leq r_{n} \leq \frac{d}{2}-n d, \quad n \geq n_{\text {infl }}\right),
\end{gathered}
$$

with amplitudes $A_{n}$ still given by Eq. (27). Sequence (47) may continue as long as the arguments of all arcosh exceed unity, i. e. as long as

$$
\frac{d}{2}+\delta \geq\left|A_{n}\right|
$$

(which is equivalent to $-\frac{d}{2}+\delta \geq\left|A_{n-1}\right|$ ). Inserting here (27), one ultimately infers the value of the reflection interval order number:

$n_{\max }^{(-)}=\left\lfloor\frac{\tau^{2} \theta_{0}^{2}-(b-\delta)^{2}+\left(\frac{d}{2}+\delta\right)^{2}}{2 \delta d}\right\rfloor \quad\left(\frac{\delta}{d}>f\left(\nu^{(-)}\right)\right)$.

The above expression is similar to Eq. (30) for positively charged particles. As one might expect, in the highenergy limit $\delta \gg d, b$ values $n_{\max }^{(+)}$and $n_{\max }^{(-)}$coincide and do not depend on the particle energy.

Expressing $t_{\text {reff }}$ from $\dot{r}\left(t_{\text {refl }}\right)=0$ and Eq. (47) in this 
case gives

$$
\begin{aligned}
& \frac{t_{\mathrm{refl}}}{\tau}=-\operatorname{arsinh} \frac{b-\delta}{A_{0}} \\
& +\sum_{n=0}^{n_{\mathrm{infl}}-1} \operatorname{arsinh} \frac{\frac{d}{2}-\delta}{A_{n}}+\sum_{n=1}^{n_{\mathrm{infl}}-1} \operatorname{arsinh} \frac{\frac{d}{2}+\delta}{A_{n}} . \\
& +\sum_{m=n_{\mathrm{infl}}}^{n_{\max }^{(-)}} \operatorname{arcosh} \frac{\frac{d}{2}+\delta}{\left|A_{m}\right|} \\
& -\sum_{m=n_{\text {infl }}}^{n_{\max }^{(-)}-1} \operatorname{arcosh} \frac{-\frac{d}{2}+\delta}{\left|A_{m}\right|} \\
& \equiv-\operatorname{arsinh} \frac{b-\delta}{\sqrt{2 \delta d\left(n_{\mathrm{infl}}-1+\nu^{(-)}\right)}} \\
& +\sum_{n=0}^{n_{\text {infl }}-1} \operatorname{arsinh} \frac{\frac{d}{2}-\delta}{\sqrt{2 \delta d\left(\nu^{(-)}+n\right)}} \\
& +\sum_{n=0}^{n_{\text {infl }}-2} \operatorname{arsinh} \frac{\frac{d}{2}+\delta}{\sqrt{2 \delta d\left(\nu^{(-)}+n\right)}}, \\
& +\sum_{n=1}^{n_{\max }^{(-)}-n_{\mathrm{infl}}+1} \operatorname{arcosh} \frac{\frac{d}{2}+\delta}{\sqrt{2 \delta d\left(n-\nu^{(-)}\right)}} \\
& -\sum_{n=1}^{n_{\max }^{(-)}-n_{\mathrm{infl}}} \operatorname{arcosh} \frac{-\frac{d}{2}+\delta}{\sqrt{2 \delta d\left(n-\nu^{(-)}\right)}} .
\end{aligned}
$$

Actually, equations (50b) can be used not only under condition (42), but also at any ratio $\frac{\delta}{d}$, provided that in capacity of $n_{\max }^{(-)}$one uses expression

$n_{\max }^{(-)}=\left\lfloor\frac{\tau^{2} \theta_{0}^{2}-(b-\delta)^{2}+\left(\frac{d}{2}-\delta\right)^{2} \Theta\left(\frac{\delta}{d}-f\left(\nu^{(-)}\right)\right)}{2 \delta d}\right\rfloor+1$

(with $\Theta(v)$ the Heavyside unit-step function) unifying (44) and (49). The universally valid formula (51) may be convenient when $t_{\text {refl }}$ is evaluated with the aid of computer for widely changing values of particle energy or crystal bending radius.

As for the physical meaning of $\nu^{(-)}$, at $\frac{\delta}{d}<f$, i. e. when inflection interval is also that of reflection, Fig. 3 illustrates that the meaning of $\nu^{(-)}$is similar to that of $\nu^{(+)}$. It is the (appropriately rescaled) kinetic transverse energy upon the particle entrance to the reflection interplanar interval, only the interval boundary now is not the atomic plane but the last potential maximum passed. In case if $\frac{\delta}{d}>f, \nu^{(-)}$does not characterize the reflection interval, and vice versa, the kinetic energy in the reflection interval is not closely related with $\nu^{(-)}$.

The obtained expressions (26,29) for the trajectory and (36, 50b) for its reflection point allow evaluating all the observables relevant to the particle passage. In the present paper, we will be interested only in the final angle of elastic reflection.

\section{Thick crystal limit (isolation of volume effects)}

Formulas (36, 50b), in principle, contain dependencies both on volume and on boundary effects. In most practical cases, the deflecting crystal may be regarded as thick, whence boundary effects are expected to turn negligible. An increase of the crystal thickness, or more precisely of the distance between the crystal boundary and the volume reflection point, may be thought of as an increase of the particle incidence angle $\theta_{0}$ (see Fig. 11). Then, it suffices to consider the limit

$$
\theta_{\text {refl }} \approx \theta_{\text {v.r. }}=2 \lim _{\theta_{0} / \theta_{c} \rightarrow \infty}\left(-\theta_{0}+\delta \frac{t_{\text {refl }}\left(\theta_{0}\right)}{\tau^{2}}\right) .
$$

With function (36), or (50b), such a limit must always be finite: indeed, at large $n_{\max }$ the sum over $n$ grows like the corresponding integral, whose asymptotic behavior straightforwardly evaluates as

$$
\begin{aligned}
\frac{t_{\mathrm{ref}}}{\tau} & \sim \int^{n_{\max }} d n\left(\frac{\frac{d}{2}+\delta}{\sqrt{2\left(\nu^{(+)}+n\right) \delta d}}+\frac{\frac{d}{2}-\delta}{\sqrt{2\left(\nu^{(+)}+n\right) \delta d}}\right) \\
& \sim \sqrt{\frac{2 n_{\max } d}{\delta}} \simeq \theta_{0} \frac{\tau}{\delta} .
\end{aligned}
$$

This leading asymptotic behavior cancels exactly the first term in (52), whilst calculation of the finite remainder requires a more accurate evaluation of the sum, which will be our task in the next section (in application to the limit $R \gg R_{c}$ ).

In general, it must be noted that function $\theta_{\text {v.r. }}(\tau, \delta, d, b)$, being a dimensionless function of 4 dimensional variables, may depend only on their 3 dimensionless ratios - say, $d / \tau, \delta / d, b / d$. At that, the last ratio is always $\sim 1$. The first one amounts to

$$
\frac{d}{\tau}=\sqrt{\frac{2 d}{R_{c}}}=2 \theta_{c}
$$

where $\theta_{c}$ is the Lindhard critical angle [8]; so, it is always small, once we are in a high-energy regime. As for the ratio

$$
\frac{2 \delta}{d}=\frac{R_{c}}{R}
$$

it may be either large or small depending on the particle energy and the crystal bending radius. The regime of particle passage through the crystal is determined solely by ratio (54).

To gain a general impression of functional dependencies involved, and to test our generic formulas 36 , 50b), let us view the dependence $\theta_{\text {v.r. }}(b)$ for different values of $R / R_{c}$. Fig. 4 shows this dependencies for positive and for negative particles. They are in fair agreement with Figs. 6 and 8 of [11]. But we will pay more attention to interpretation of the features observed in the figures:

1. The origin of the recurrent structure in variable $b$ with a tapering period is, obviously, due to $\theta_{\text {v.r. }}$ dependence on $b$ through $\nu^{( \pm)}$alone (see Eqs. (35), 


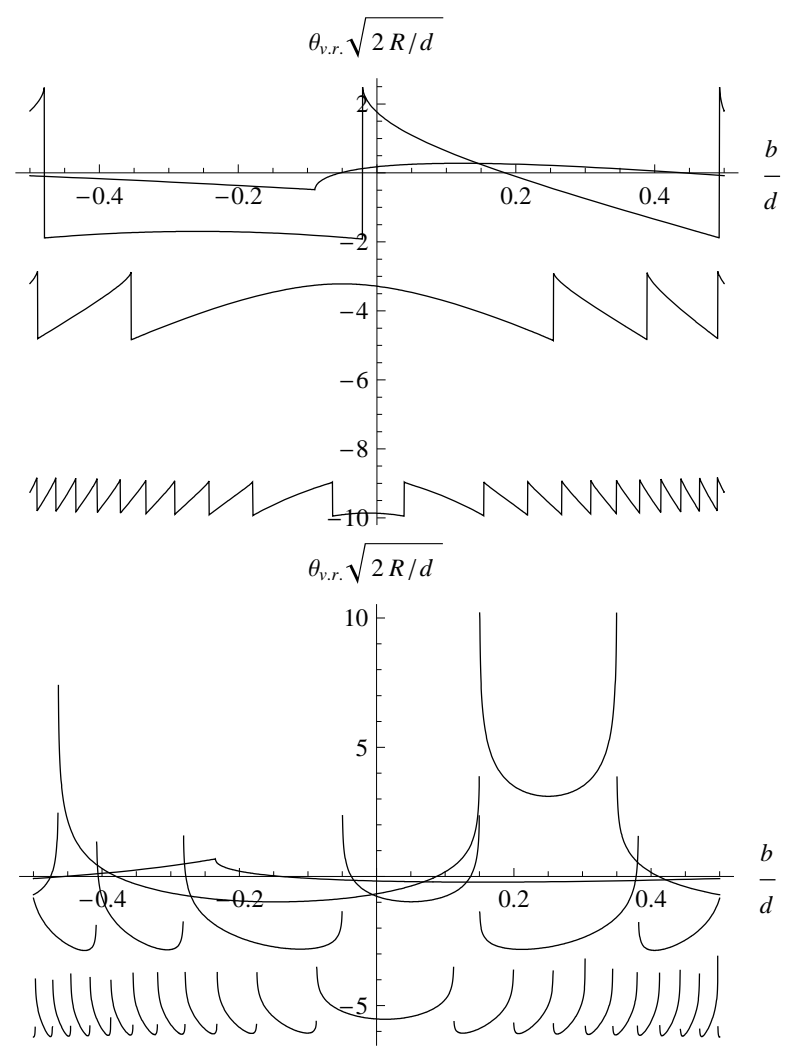

FIG. 4: The volume reflection angle $\theta_{\text {v.r. }}$ as a function of impact parameter $b$, for $R / R_{c}=1 / 3,2,10,40$. Top panel for positively charged particles, bottom panel - for negatively charged particles. Beyond the shown unit interval the picture repeats periodically.

(40)), insofar as $\nu^{( \pm)}$, involving an operation of fractional part, is a periodic function of $\frac{(b \pm \delta)^{2}}{2 \delta d}$, which in the interval $-\frac{d}{2}<b<\frac{d}{2}$ makes $\sim 2 \frac{d^{2}}{8 \delta d}=$ $\frac{R}{2 R_{c}} \gg 1$ periods. Also, since $\nu^{( \pm)}$is an even function of $b \pm \delta$, the particle deflection angle is a symmetric function of $b$ with respect to point $b=-\delta$ for positively charged particles, and with respect to $b=\delta$ for negative particles.

2. Another feature of $\theta_{\text {v.r. }}(b)$ dependencie(s) is that, for negatively charged particles, the reflection angle blows up (formally) to $+\infty$ at certain values of impact parameters. Physically, that corresponds to close matching of the particle transverse energy to the height of a (locally parabolic) effective potential barrier - the situation known as orbiting (see [12]) 27]. The asymptotics of the divergences is logarithmic [12], as follows from the general integral expression of the deflection angle in a central potential $V(r)[28]$ :

$$
\begin{aligned}
& \theta \underset{E \gg m}{\approx} 2 M \int_{r_{\min }}^{\infty} \frac{d r /(R+r)^{2}}{\sqrt{(E-V(r))^{2}-M^{2} /(R+r)^{2}}}-\pi \quad(55 \mathrm{a}) \\
& \underset{R \gg r, b}{\approx} \frac{1}{R} \int_{r_{\min }}^{\infty} \frac{d r}{\sqrt{\frac{\theta_{0}^{2}}{4}+\frac{V_{\text {eff }}(b)-V_{\text {eff }}(r)}{2 E}}}-\pi \\
& \underset{\text { orbit. }}{\sim} \theta_{c} \frac{4 \delta}{d} \int_{\left|r-r_{\text {saddle }}\right| \lesssim d} \frac{d r}{\sqrt{\frac{\Delta E_{\perp}}{\left|F_{\text {max }}\right|}+\left(r-r_{\text {saddle }}\right)^{2}}}+\ldots(55 \mathrm{c}) \\
& \simeq \theta_{c} \frac{4 \delta}{d}\left\{\begin{array}{l}
\ln \frac{1}{\nu^{(-)}+\ldots}\left(\triangle E_{\perp} \propto \nu^{(-)} \rightarrow+0\right) \\
\frac{1}{2} \ln \frac{1}{1-\nu^{(-)}}+\ldots\left(\triangle E_{\perp} \propto \nu^{(-)}-1 \rightarrow-0\right) .
\end{array}\right.
\end{aligned}
$$

Here

$$
M \simeq(R-b)[E-V(b)]\left(1-\theta_{0}^{2} / 2\right)
$$

is the particle angular momentum relative to the crystal bend axis,

$$
V_{\text {eff }}(r)=V(r)-E \frac{r}{R}
$$

is the effective potential including the centrifugal energy, $r_{\text {saddle }}$ - the position of maximum of the effective potential barrier whose height in the case of orbiting happens to be close to the particle energy, and $\triangle E_{\perp}-$ the transverse energy variation relative to the height of the effective potential barrier. The factor $\frac{1}{2}$ in the $\triangle E_{\perp}<0$ alternative of Eq. (55d) arises because the integration in (55c) is then carried out only over the one-sided neighborhood of $r_{\text {saddle }}$ where the radicand stays positive (see Fig. 馬).

3. It must be noticed that for negative particles function $\theta_{\text {v.r. }}(b)$ has smooth minima, which must correspond to caustics, i. e., to rainbow scattering [12].

4. In contrast, for positive particles the potential in its maximum is not differentiable, excluding both orbiting and rainbow scattering for this case. With some smearing of the potential around the atomic planes, these affects, of course, re-appear.

The proper question is whether it is possible to derive at least the particle final deflection angle (related with $\frac{t_{\text {refl }}}{\tau}$ ) from the more conventional integral representation approach [2, 9]. In that approach momentum and transverse energy conservation laws are incorporated automatically, so there is no need to connect trajectories on the interval borders. Indeed, specializing in (56, 55b)

$$
V(r)=F_{\max } d\left(\frac{r}{d}+n\right)^{2}, \quad n=-\left\lfloor\frac{r}{d}+\frac{1}{2}\right\rfloor
$$

(at $r<0 n \geq 0$ ) 


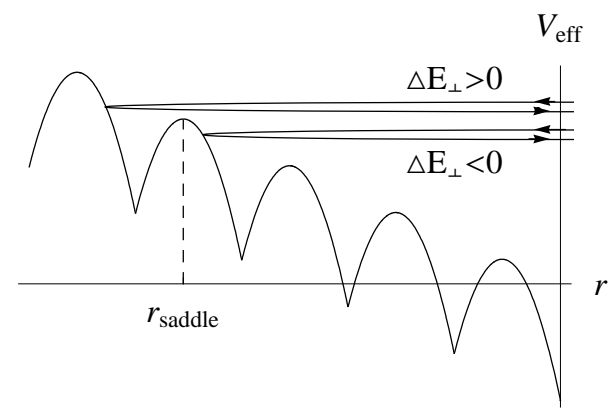

FIG. 5: The relation between the particle transverse energy and the effective potential energy $V_{\text {eff }}$ (including the centrifugal potential) under the conditions of negatively charged particle orbiting in a bent crystal. The leading logarithmic contribution to integral (55b) comes from the vicinity of point $r_{\text {saddle }}$ - the coordinate of the effective potential maximum to which the particle transverse energy happens to be close. In the case $\Delta E_{\perp}>0$ the particle sweeps the two-sided neighborhood of $r_{\text {saddle, whereas at }} \triangle E_{\perp}<0$ - its one-sided neighborhood only.

and using basic integral

$$
\begin{aligned}
\int_{-d / 2-n d}^{d / 2-n d} & \frac{d r}{\sqrt{\tau^{2} \theta_{0}^{2}+b^{2}+2 \delta(b+r)-(r+n d)^{2}}} \\
& =\arcsin \frac{\frac{d}{2}-\delta}{\sqrt{\tau^{2} \theta_{0}^{2}+(b+\delta)^{2}-2 n \delta d}} \\
& +\arcsin \frac{\frac{d}{2}+\delta}{\sqrt{\tau^{2} \theta_{0}^{2}+(b+\delta)^{2}-2 n \delta d}}
\end{aligned}
$$

for positively charged particles, and a similar one for negative particles, we reproduce the inverse trigonometric and hyperbolic functions encountered in (34, 46, 50a). But the integral representation approach wouldn't give us explicit trajectories $r(t)$ (rather, $t(r)$, to be solved for $r$ ), and the geometric interpretation (Figs. 20 3).

On the other hand, from the integral representation for the final angle we might derive the result in form of a sum of analytic functions also for a more complicated parametrization of the inter-planar potential - e. g., adding thereto a term proportional to $r^{4}$. Then instead of arcsines one would get elliptic functions. But it is the simplicity of functions in the sum that permits us, in the important limit $R \gg R_{c}$, when the number of terms in the sum gets large, to replace the sums by integrals and do them in closed form. In this sense, analytic investigation only begins here.

\section{VOLUME REFLECTION REGIME (MODERATELY HIGH ENERGIES, $R \gg R_{c}$ )}

As we had mentioned in the Introduction, and as Fig. 4 does confirm, under the condition $R \gg R_{c}$, i. e. $2 \delta \ll d$, the particle deflection angles depends weakly on the impact parameter. So, it is interesting, in the first place, to determine the numerical value of the limiting ratio $\lim _{R / R_{c} \rightarrow \infty} \frac{\theta_{\mathrm{v} . \mathrm{r}}}{\theta_{c}}$. Secondly, it is desirable to determine the final beam shape and quantify its angular width as a function of $R / R_{c}$. That will be our aim for the present section. The treatment is somewhat different for the cases of positively and negatively charged particles, because of the difference between the functional form of initial Eqs. (36) and (50b).

\section{A. Positive particles}

For positively charged particles, in the considered limiting case $\delta \ll d$, say, quantity $\left(\frac{d}{2} \pm \delta\right)^{2}+2\left(\nu^{(+)}+n\right) \delta d$ entering the denominators in (36a) varies relatively little as $n$ changes from $n$ to $n+1$. Thus it must be possible to replace the summation in (36) by integration. The proper mathematical tool for that is the Euler-Maclaurin formula (see, e. g., [17]) which reads

$$
\sum_{n=0}^{N} f(n)=\frac{1}{2} f(0)+\int_{0}^{N} d n f(n)+\frac{1}{2} f(N)+\mathcal{O}\left(\frac{d f}{d n}\right) .
$$

Employing this formula for approximation of each of the sums in (36b) (which is somewhat more convenient than Eq. (36a)), one gets [29]

$$
\begin{aligned}
& \sum_{n=0}^{n_{\max }^{(+)}-1} \operatorname{arccot} \frac{\sqrt{2 \delta d\left(\nu^{(+)}+n\right)}}{\frac{d}{2} \pm \delta} \\
& =\frac{1}{2} \operatorname{arccot} \frac{\sqrt{2 \delta d \nu^{(+)}}}{\frac{d}{2} \pm \delta}+\int_{\nu^{(+)}}^{n_{\max }^{(+)}+\nu^{(+)}-1} d n \operatorname{arccot} \frac{\sqrt{2 \delta d n}}{\frac{d}{2} \pm \delta} \\
& +\frac{1}{2} \operatorname{arccot} \frac{\sqrt{2 \delta d\left(n_{\max }^{(+)}+\nu^{(+)}-1\right)}}{\frac{d}{2} \pm \delta}+\mathcal{O}\left(\sqrt{\frac{\delta}{d}}\right),
\end{aligned}
$$

where we had estimated, for all $n$,

$$
\left|\frac{d}{d n} \operatorname{arccot} \frac{\sqrt{2 \delta d\left(\nu^{(+)}+n\right)}}{\frac{d}{2} \pm \delta}\right| \lesssim \sqrt{\frac{\delta}{d}} .
$$

The two end-point contributions in the third line of (60) are small as $\mathcal{O}(\delta / d)$ relative to the integral, but still they need to be kept if we wish to describe not only the mean deflection, but also the scattered beam shape.

Taking the indefinite integral in Eq. (60) by parts

$$
\int d n \operatorname{arccot} \sqrt{a n}=\frac{1}{a}[(1+a n) \operatorname{arccot} \sqrt{a n}+\sqrt{a n}],
$$


one brings (60) to the form

$$
\begin{aligned}
& \sum_{n=0}^{n_{\max }^{(+)}-1} \operatorname{arccot} \frac{\sqrt{2 \delta d\left(\nu^{(+)}+n\right)}}{\frac{d}{2} \pm \delta} \\
& \underset{\delta \ll d}{\approx} \frac{\left(\frac{d}{2} \pm \delta\right)^{2}}{2 \delta d}\left(\left[1+\frac{2 \delta d}{\left(\frac{d}{2} \pm \delta\right)^{2}}\left(n_{\max }^{(+)}+\nu^{(+)}-1\right)\right]\right. \\
& \cdot \operatorname{arccot} \frac{\sqrt{2 \delta d\left(n_{\max }^{(+)}+\nu^{(+)}-1\right)}}{\frac{d}{2} \pm \delta} \\
& +\frac{\sqrt{2 \delta d\left(n_{\max }^{(+)}+\nu^{(+)}-1\right)}}{\frac{d}{2} \pm \delta} \\
& \left.-\left[1+\frac{2 \delta d \nu^{(+)}}{\left(\frac{d}{2} \pm \delta\right)^{2}}\right] \operatorname{arccot} \frac{\sqrt{2 \delta d \nu^{(+)}}}{\frac{d}{2} \pm \delta}-\frac{\sqrt{2 \delta d \nu^{(+)}}}{\frac{d}{2} \pm \delta}\right) \\
& +\frac{1}{2} \operatorname{arccot} \frac{\sqrt{2 \delta d \nu^{(+)}}}{\frac{d}{2} \pm \delta}+\frac{1}{2} \operatorname{arccot} \frac{\sqrt{2 \delta d\left(n_{\max }^{(+)}+\nu^{(+)}-1\right)}}{\frac{d}{2} \pm \delta} \\
& +\mathcal{O}\left(\sqrt{\frac{\delta}{d}}\right) \text {. }
\end{aligned}
$$

In the limit $n_{\max }^{(+)} \rightarrow \infty$, with the use of asymptotic expansion $\operatorname{arccot} \sqrt{\eta}=\frac{\pi}{2}-\sqrt{\eta}+\mathcal{O}\left(\eta^{3 / 2}\right)$, expression (61) reduces to

$$
\begin{aligned}
& \sum_{n=0}^{n_{\max }^{(+)}-1} \operatorname{arccot} \frac{\sqrt{2 \delta d\left(\nu^{(+)}+n\right)}}{\frac{d}{2} \pm \delta} \\
& \underset{n_{\max }^{(+)} \gg 1}{\rightarrow} \frac{d \pm 2 \delta}{\sqrt{2 \delta d}} \sqrt{n_{\max }^{(+)}} \\
& -\frac{\left(\frac{d}{2} \pm \delta\right)^{2}}{2 \delta d}\left[\left(1+\frac{2 \delta d}{\left(\frac{d}{2} \pm \delta\right)^{2}}\right)\left(\frac{\pi}{2}-\frac{\sqrt{2 \delta d \nu^{(+)}}}{\frac{d}{2} \pm \delta}\right)+\frac{\sqrt{2 \delta d \nu^{(+)}}}{\frac{d}{2} \pm \delta}\right] \\
& +\frac{1}{2} \cdot \frac{\pi}{2}+\mathcal{O}\left(\frac{1}{\sqrt{n_{\max }^{(+)}}}, \sqrt{\frac{\delta}{d}}\right) .
\end{aligned}
$$

Here, one notices that terms $-\frac{\sqrt{2 \delta d \nu(+)}}{\frac{d}{2} \pm \delta},+\frac{\sqrt{2 \delta d \nu(+)}}{\frac{d}{2} \pm \delta}$ in the brackets in (62) cancel. Further on, inserting (62) to (36b) and this to (52), we witness the anticipated cancelation of the large terms $-\theta_{0}+\frac{\sqrt{2 \delta d}}{\tau} n_{\max }^{(+)} \cong 0$, and

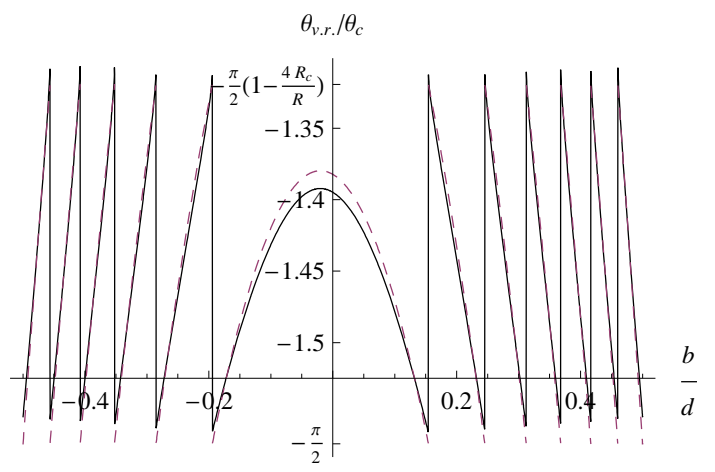

FIG. 6: Solid curve - positively charged particle reflection angle $\theta_{\text {v.r. }}$ versus impact parameter $b$, for $R / R_{c}=25$. Dashed curve - approximation (63). The central segment of the curves is strongly $\theta_{0}$-dependent.

ultimately arrive at result

$$
\begin{aligned}
\theta_{\text {v.r. }}(b) \approx & -2 \theta_{0}+2 \frac{\delta}{\tau}\left(\frac{\pi}{2}+\frac{2 d}{\sqrt{2 \delta d}} \sqrt{n_{\max }^{(+)}}\right. \\
& -\frac{\pi}{2}\left[\frac{\left(\frac{d}{2}+\delta\right)^{2}}{2 \delta d}+\nu^{(+)}\right]+\frac{\pi}{4} \\
& \left.-\frac{\pi}{2}\left[\frac{\left(\frac{d}{2}-\delta\right)^{2}}{2 \delta d}+\nu^{(+)}\right]+\frac{\pi}{4}\right) \\
\equiv & -\frac{\pi}{2} \theta_{c}\left[1-\frac{4 R_{c}}{R}\left(1-\nu^{(+)}(b)\right)+\mathcal{O}\left(\frac{R_{c}^{3 / 2}}{R^{3 / 2}}\right)\right]
\end{aligned}
$$

(with $\nu^{(+)}(b)$ being given by Eq. (35)).

Comparison of approximation (63) with the exact result (36) is shown in Fig. 6. (Actually, the given approximation appears to be numerically accurate starting from $R / R_{c} \sim 5$ ). From the figure (or Eq. (63)) one concludes that in the first approximation all the particles are deflected to the same angle $\approx-\frac{\pi}{2} \theta_{c}$. There is also some dispersal of the scattering angles, depending on the particle impact parameter, of the full width

$$
\triangle \theta_{\text {v.r. }}=\theta_{c} \frac{2 \pi R_{c}}{R} \equiv \frac{2 \pi \delta}{\tau} . \quad \text { (posit. charged particles) }
$$

The observable quantity, however, is not the indicatrix $\theta_{\text {v.r. }}(b)$ but the scattering differential cross-section (final particle flux averaged over the impact parameters $b$ ) as a function of the scattering angle $\theta_{v \text { v.r. }}$. Therefore, it is desirable to reconstruct the latter dependence issuing from the first. That does not pose any principal problem, granted the linearity of dependence $\theta_{\text {v.r. }}\left(\nu^{(+)}\right)$.

\section{Differential cross-section}

Turning to evaluation of the differential cross-section, one encounters a certain complication: the $b$-dependent 
quantity $\nu^{(+)}$in (63) also contains dependence on $\theta_{0}$. The fact of residual $\theta_{0}$-dependence was noticed in 11 . To some degree, it conflicts with our initial assumption about the boundary condition vanishing influence in the limit of large $\theta_{0} / \theta_{c}$. We can not revoke it at the present stage, since in equation (52) we had already incorporated the facilitating assumption of the trajectory symmetry with respect to point $t_{\text {refl }}$. Obviously, the sensitivity to the boundary conditions in general destroys such a symmetry. Furthermore, $\theta_{\text {refl }}$ might as well contain a dependence on the particle exit angle relative to the atomic planes, which we did not even take trouble to specify. Altogether, that may rise a suspicion that the $b$-dependent correction obtained in (63) is unreliable for evaluating the differential cross-section. Fortunately, the impediment is not fatal and can be overcome within the present framework. In principle, the differential crosssection sensitivity to $\theta_{0}$ is attenuated with the increase of $R / R_{c}$, but more importantly, we will prove that upon averaging over a tiny interval of $\theta_{0}$ this dependence is eliminated completely.

To begin with, the differential cross-section involves only a derivative of function $\theta_{\text {v.r. }}(b)$ :

$$
\begin{aligned}
\frac{d \lambda}{d \theta_{\text {v.r. }}} & =\sum_{m} \frac{1}{\left|d \theta_{\text {v.r. }} / d b\right|_{b=b_{m}\left(\theta_{\text {v.r. }}\right)}} \\
& \equiv \frac{R}{2 \pi \theta_{c} R_{c}} \sum_{m} \frac{1}{\left|d \nu^{(+)} / d b\right|_{b=b_{m}\left(\nu(+)\left(\theta_{\text {v.r. } .}\right)\right.}},
\end{aligned}
$$

where $b_{m}(\theta)$ is the set of all the roots of equation $\theta_{\mathrm{v} . r .}(b)=\theta$ belonging to the interval $-\frac{d}{2}<b<\frac{d}{2}$. Now, at $R / R_{c} \gg 1$ the number of roots $b_{m}$ of equation $\theta_{\text {v.r. }}=\theta$ is large, and so, in general, they are densely distributed over the finite definition interval $-\frac{d}{2}<b<\frac{d}{2}$. It appears that the root distribution density is just proportional to the derivative in the denominator of (65) (the formal demonstration is relegated to the Appendix). Therefore, the sum appearing in (65) approximately equals to just the $b$ variation interval length, i. e., $d$. However, the relation expected thereby,

$$
\frac{d \lambda}{d \theta_{\mathrm{v} . \mathrm{r} .}} \simeq \frac{R d}{2 \pi \theta_{c} R_{c}}
$$

does not yet hold uniformly in $b$, and hence in $\theta_{\text {v.r. }}$ For instance, in a neighborhood of point $b=-\delta$ we have in the denominator of (65) $\partial \nu^{(+)} / \partial b \rightarrow 0$ (see Fig. 6), so there the differential cross-section blows up above the plateau (66) (see Appendix). But the latter peak position on the $\theta_{\text {v.r. }}$ axis depends sharply on the value of $\theta_{0}$ and hence is essentially "random", needing to be averaged over.

Indeed, one notices that the dependence of $\nu^{(+)}$on $\theta_{0}$ is quadratic, so a situation is possible when the incident particle beam divergence is smaller than the angular spread acquired in the crystal:

$$
\triangle \theta_{0} \ll \triangle \theta_{\text {v.r. }}
$$

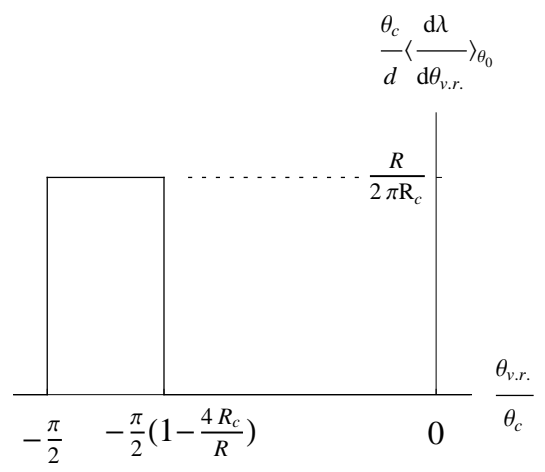

FIG. 7: Asymptotic (at $R \gg R_{c}$ ) behavior of the $\theta_{0}$-averaged differential cross-section for positively charged particle scattering (Eq. (71)). The area under the rectangular curve is unity, representing the total probability. In higher orders in $R_{c} / R$ the distribution edges must smear out (see discussion in the text).

but at the same time, the indeterminance of $\frac{\tau^{2} \theta_{0}^{2}}{2 \delta d} \approx n_{\max }^{(+)}$ is greater than unity:

$$
\triangle\left(\frac{\tau^{2} \theta_{0}^{2}}{2 \delta d}\right)=\frac{\tau^{2} \theta_{0}}{\delta d} \triangle \theta_{0} \gg 1 .
$$

Together, Eqs. (67, 68) may be viewed as a double inequality

$$
\frac{2 \delta}{\tau} \frac{\theta_{c}}{\theta_{0}} \ll \triangle \theta_{0} \ll \frac{2 \pi \delta}{\tau} \quad\left(\theta_{0}-\text { averaging }\right) .
$$

Here the sufficient gap exists provided

$$
\theta_{0} \ggg \frac{\theta_{c}}{\pi} .
$$

This is basically the same condition that we had assumed in writing Eq. (52), thus for derivation of a $\theta_{0}$-averaged differential cross-section we can safely rely on Eq. (63).

Ultimately, we can make a specific statement that under conditions (69), upon $\theta_{0}$-averaging, the differential cross-section equals to constant (66) over an interval where roots $b_{m}$ exist. This interval is determined in the Appendix (Eq. (theta-interval)). So, the $\theta_{0}$-averaged differential cross-section (the final beam profile) is described by a simple rectangular function

$$
\begin{aligned}
&\left\langle\frac{d \lambda}{d \theta_{\text {v.r. }}}\right\rangle_{\theta_{0}} \approx \frac{R d}{2 \pi \theta_{c} R_{c}} \\
& \cdot \Theta\left(\theta_{\text {v.r. }}+\frac{\pi}{2} \theta_{c}\right) \Theta\left(-\theta_{\text {v.r. }}-\frac{\pi}{2} \theta_{c}\left(1-\frac{4 R_{c}}{R}\right)\right)
\end{aligned}
$$

(see Fig. 7).

\section{Comparison with experiment}

The deflection angle mean value is least affected by multiple scattering, and thus, may be directly compared 
with the experiment. From (63) we obviously infer

$$
\left\langle\theta_{\text {v.r. }}\right\rangle=-\frac{\pi}{2} \theta_{c}\left(1-\frac{2 R_{c}}{R}\right) \equiv-\frac{\pi}{2} \sqrt{\frac{d}{2 R_{c}}}\left(1-\frac{2 R_{c}}{R}\right) .
$$

The property of (72) is the linearity of dependence on the crystal curvature $R^{-1}$; the linear kind of dependence was indeed noticed in CERN experiments with $E=400 \mathrm{GeV} \mathrm{GeV} / \mathrm{cm}$ [14].

To make quantitative comparison with the experiment, one needs to specify the potential strength in our model. In reality, the $\mathrm{Si}$ (110) inter-planar potential is characterized by 2 parameters: $F_{\max } \approx 6 \mathrm{GeV} / \mathrm{cm}$ (usually used for evaluation of $R_{c}$ for channeling processes) and the well depth $V_{0}=22.7 \mathrm{eV}$ (usually used for evaluation of the critical angle $\theta_{c}$ for volume reflection). The relation $V_{0}=\frac{1}{4} F_{\max } d$ implied by a quadratic potential model only holds with accuracy $\approx 20 \%$ :

$$
\begin{gathered}
22.7 \mathrm{eV}=V_{0} \neq \frac{1}{4} F_{\max } d=28.8 \mathrm{eV} \\
(d=1.92 \AA \text { for } \mathrm{Si}(110)) .
\end{gathered}
$$

If we evaluate $R_{c}$ in (72) as $R_{c}=E / F_{\max }=0.67 \mathrm{~m}$, it will produce too large $\left|\left\langle\theta_{\mathrm{v} . \mathrm{r} .}\right\rangle\right|$. But evaluating both $R_{c}$ and $\theta_{c}$ as $R_{c}=\frac{E d}{4 V_{0}} \approx 0.85 \mathrm{~m}, \theta_{c}=\sqrt{2 V_{0} / E} \approx 11 \mu \mathrm{rad}$, and substituting to Eq. (72), we get a satisfactory agreement with the experiment (see Fig. 8).

As for the obtained rectangular profile shape, it is more sensitive to multiple scattering, and was not yet probed by experiments (the optimal experimental conditions will be specified in Sec. VI). But we can compare our profile with the available numerical simulation results using a realistic, smeared potential, without multiple scattering: [11], Fig. 6. In that case, the positive particle profile shows indeed a signature of flattening ("shoulder") but near its edges the distribution behaves differently, exhibiting a subtle divergence (rainbow) at the outer edge, and decreasing continuously on the inward side. So, for positively charged particles our simplified model of parabolic inter-planar potential describes the final beam profile only quantitatively, though it is able to predict the distribution width and mean value.

Next, turning to the negative particle reflection problem, we shall see that in this case our analytic approach is able to capture also the final beam profile edge details.

\section{B. Negative particles}

In the case of negative particles, the starting point is Eq. (46) (relevant under (strong) condition (42)), and again, it has to be examined with the object to trade the sum for an integral. First of all, it has to be minded that at $n \sim 1$ the hyperbolic arcsine arguments vary significantly, but at the same time they are large, whereas hyperbolic arcsine of a large argument is close to logarithm of a large argument: $\operatorname{arsinh} v \underset{v \gg 1}{\widetilde{N}} \ln 2 v$, and thus

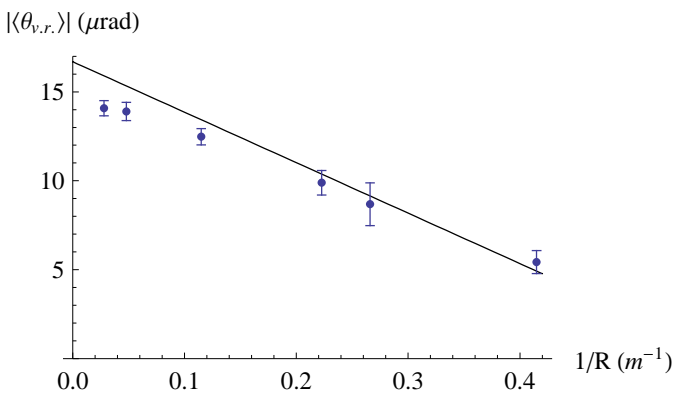

FIG. 8: Mean volume reflection angle dependence on the crystal curvature, at $E=400 \mathrm{GeV}$, for silicon crystal in orientation (110). Points - experimental data from [14]. The line prediction of Eq. (72), with parameters evaluated as explained in the text.

varies relatively little. On the other hand, in the domain of large $n$ the arguments of the arcsines vary little. Therefore, over the entire summation interval both sums involved may be approximated via integrals. Yet, first terms in the sums are singular functions of $\nu^{(-)}$, and therefore are better taken into account separately. Thereby, application of the Euler-Maclaurin formula to the first of the sums in Eq. (46) gives

$$
\begin{aligned}
& \sum_{n=0}^{n_{\text {infl }}-1} \operatorname{arsinh} \frac{\frac{d}{2}-\delta}{\sqrt{2 \delta d\left(\nu^{(-)}+n\right)}} \\
& \approx \ln \frac{d-2 \delta}{\sqrt{2 \delta d \nu^{(-)}}}+\frac{1}{2} \ln \frac{d-2 \delta}{\sqrt{2 \delta d\left(\nu^{(-)}+1\right)}} \\
& +\int_{\nu^{(-)}+1}^{\nu^{(-)}+n_{\text {infl }}-1} d n \operatorname{arsinh} \frac{\frac{d}{2}-\delta}{\sqrt{2 \delta d} \sqrt{n}} \\
& +\frac{1}{2} \operatorname{arsinh} \frac{\frac{d}{2}-\delta}{\sqrt{2 \delta d\left(\nu^{(-)}+n_{\text {infl }}-1\right)}} .
\end{aligned}
$$

The next-to-leading order (derivative-related) correction term [17] to (73) amounts

$$
\left.\frac{1}{12} \frac{d}{d n} \operatorname{arsinh} \frac{\frac{d}{2}-\delta}{\sqrt{2 \delta d\left(\nu^{(-)}+n\right)}}\right|_{n=1} ^{n=n_{\mathrm{infl}}-1} \approx-\frac{1}{24\left(1+\nu^{(-)}\right)} .
$$

We will omit it because of the smallness of the numerical coefficient $\frac{1}{24}$, although, in principle, asymptotically it is also relevant (the same is true for all the higher derivatives, whose contributions enter with yet smaller coefficients (involving inverse factorial and Bernoulli numbers)).

Next, calculating the indefinite integral in Eq. (73) by parts,

$$
\int d n \operatorname{arsinh} \frac{a}{\sqrt{n}}=n \operatorname{arsinh} \frac{a}{\sqrt{n}}+a \sqrt{a^{2}+n},
$$


we bring (73) to form

$$
\begin{aligned}
& \sum_{n=0}^{n_{\text {infl }}-1} \operatorname{arsinh} \frac{\frac{d}{2}-\delta}{\sqrt{2 \delta d} \sqrt{\nu^{(-)}+n}} \\
& \approx\left(\nu^{(-)}+n_{\text {infl }}-1\right) \operatorname{arsinh} \frac{\frac{d}{2}-\delta}{\sqrt{2 \delta d\left(\nu^{(-)}+n_{\text {infl }}-1\right)}} \\
& +\frac{\frac{d}{2}-\delta}{\sqrt{2 \delta d}} \sqrt{\frac{\left(\frac{d}{2}-\delta\right)^{2}}{2 \delta d}+\nu^{(-)}+n_{\text {infl }}-1} \\
& -\left(1+\nu^{(-)}\right) \operatorname{arsinh} \frac{\frac{d}{2}-\delta}{\sqrt{2 \delta d\left(1+\nu^{(-)}\right)}} \\
& -\frac{\frac{d}{2}-\delta}{\sqrt{2 \delta d}} \sqrt{\frac{\left(\frac{d}{2}-\delta\right)^{2}}{2 \delta d}+1+\nu^{(-)}+\ln \frac{d-2 \delta}{\sqrt{2 \delta d \nu^{(-)}}}} \\
& +\frac{1}{2} \ln \frac{d-2 \delta}{\sqrt{2 \delta d\left(1+\nu^{(-)}\right)}}+\frac{1}{2} \operatorname{arsinh} \frac{\frac{d}{2}-\delta}{\sqrt{2 \delta d\left(\nu^{(-)}+n_{\text {infl }}-1\right)}}
\end{aligned}
$$

Now, in the thick-crystal limit $n_{\text {infl }} \rightarrow \infty$, Eq. (76) simplifies to

$$
\begin{aligned}
& \sum_{n=0}^{n_{\text {infl }}-1} \operatorname{arsinh} \frac{\frac{d}{2}-\delta}{\sqrt{2 \delta d} \sqrt{\nu^{(-)}+n}} \\
& \underset{n_{\text {infl }} \gg 1}{\rightarrow} \sqrt{n_{\text {inf }}} \frac{d-2 \delta}{\sqrt{2 \delta d}}-\left(1+\nu^{(-)}\right) \ln \frac{d-2 \delta}{\sqrt{2 \delta d\left(1+\nu^{(-)}\right)}} \\
& -\frac{\left(\frac{d}{2}-\delta\right)^{2}}{2 \delta d} \sqrt{1+\frac{2 \delta d}{\left(\frac{d}{2}-\delta\right)^{2}}\left(1+\nu^{(-)}\right)}+\ln \frac{d-2 \delta}{\sqrt{2 \delta d \nu^{(-)}}} \\
& \quad+\frac{1}{2} \ln \frac{d-2 \delta}{\sqrt{2 \delta d\left(1+\nu^{(-)}\right)}} \\
& \cong \sqrt{n_{\text {infl }}} \frac{d-2 \delta}{\sqrt{2 \delta d}}-\left(\frac{1}{2}+\nu^{(-)}\right) \ln \frac{d-2 \delta}{\sqrt{2 \delta d\left(1+\nu^{(-)}\right)}} \\
& \quad-\frac{\left(\frac{d}{2}-\delta\right)^{2}}{2 \delta d}-\frac{1}{2}\left(1+\nu^{(-)}\right)+\ln \frac{d-2 \delta}{\sqrt{2 \delta d \nu^{(-)}}} .
\end{aligned}
$$

Similarly, the second term in (46) reduces to

$$
\begin{aligned}
& \sum_{n=0}^{n_{\text {infl }}-2} \operatorname{arsinh} \frac{\frac{d}{2}+\delta}{\sqrt{2 \delta d\left(\nu^{(-)}+n\right)}} \\
& \rightarrow \sqrt{n_{\text {inf }}} \frac{d+2 \delta}{\sqrt{2 \delta d}}-\left(\frac{1}{2}+\nu^{(-)}\right) \ln \frac{d+2 \delta}{\sqrt{2 \delta d\left(1+\nu^{(-)}\right)}} \\
& \quad-\frac{\left(\frac{d}{2}+\delta\right)^{2}}{2 \delta d}-\frac{1}{2}\left(1+\nu^{(-)}\right)+\ln \frac{d+2 \delta}{\sqrt{2 \delta d \nu^{(-)}}}
\end{aligned}
$$

Inserting (77) and (78) to Eqs. (46) and thereupon to (52), after a simple rearrangement one is left with the

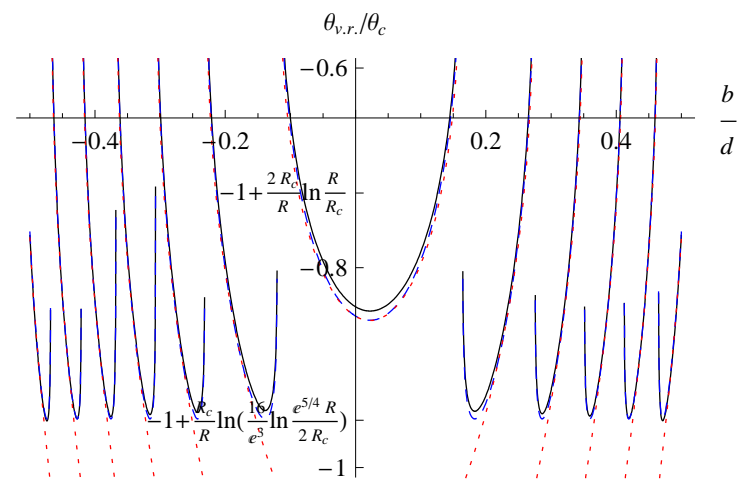

FIG. 9: Comparison of the exact formula (52 46) for the negatively charged particle indicatrix (black solid curve) with the approximation (79) (blue dashed curve) and approximation (89) (red dotted curve) for $R / R_{c}=25$. The central segment of the curves is strongly $\theta_{0}$-dependent.

final result

$$
\begin{gathered}
\theta_{\mathrm{v} . \mathrm{r} .} \approx-\theta_{c}\left[1-\frac{2 R_{c}}{R}\left(\frac{1}{2} \ln \frac{1}{e\left(1-\nu^{(-)}\right)}+\ln \frac{1}{\nu^{(-)}}\right.\right. \\
\left.\left.-\left(\nu^{(-)}+\frac{1}{2}\right) \ln \frac{e}{\left(1+\nu^{(-)}\right)}+\left(1-\nu^{(-)}\right) \ln \frac{R}{R_{c}}\right)\right]
\end{gathered}
$$

(the definition for $\nu^{(-)}(b)$ is Eq. (40)). Note that logarithmic asymptotics of this expression at $\nu^{(-)} \rightarrow 0$ and at $\nu^{(-)} \rightarrow 1$ agrees with the general law (55d).

The exact (52) vs. approximate (79) expressions for the indicatrix $\theta_{\text {v.r. }}\left(\nu^{(-)}(b)\right)$ are compared in Fig. 9 .

\section{Differential cross-section}

To deduce the observable differential cross-section from the available indicatrix, we have again to issue from Eq. (65). To some extent, the same procedure as for positively charged particles applies here, leading to representation

$$
\frac{d \lambda}{d \theta_{\text {v.r. }}}=\left.\sum_{j=1}^{2}\left|\frac{d \nu^{(-)}}{d \theta_{\text {v.r. }}}\right| \sum_{m} \frac{1}{\left|\partial \nu_{j}^{(-)} / \partial b\right|}\right|_{b=b_{m}\left(\nu_{j}^{(-)}\left(\theta_{\text {v.r. })}\right)\right.},
$$

where $b_{m}\left(\nu^{(-)}\right)$is the solution of Eq. (40), $\sum_{j=1}^{2}$ accounts for the existence of two roots for equation $\theta_{\text {v.r. }}=$ $\theta_{\text {v.r. }}\left(\nu^{(-)}\right)$with the function (79). Again, upon averaging over $\theta_{0}$ under condition (69) (cf. Appendix), one obtains:

$$
\frac{1}{d}\left\langle\frac{d \lambda}{d \theta_{\mathrm{v} . \mathrm{r} .}}\right\rangle_{\theta_{0}}=\sum_{j=1}^{2}\left|\frac{d \nu_{j}^{(-)}}{d \theta_{\mathrm{v} . \mathrm{r} .}}\right|,
$$

i. e., averaging over $b$ (and a tiny interval of $\theta_{0}$ ) reduces to averaging over $\nu^{(-)}$(transverse energy). Note that when $\nu^{(-)}$becomes a uniformly distributed random quantity in 
a unit variation interval, for each given $b$, and thus $\theta_{\text {v.r., }}$, one can always unambiguously tell whether $\nu^{(-)}=\nu_{1}^{(-)}$ or $\nu^{(-)}=\nu_{2}^{(-)}$, so the probability normalization is conserved under conditions of summation over the branches.

A technical distinction of the case with negative particles is that equation (79) can not be resolved with respect to $\nu^{(-)}$in an explicit and exact form. Of course, it can be easily solved numerically; the differential cross-section so evaluated is shown in Fig. 10, by a solid curve. On the other hand, it is also useful to pursue an analytic but approximate approach, based on different approximations in different regions of $\nu^{(-)}$, to which we yet pay some labor.

\section{Asymptotic evaluation of the final beam profile}

In Eq. (79) at typical $\nu^{(-)}$the leading term is the last one, where $\nu^{(-)}$is multiplied by a large logarithm. Besides that, in the domain of $\nu^{(-)}$close to 1 the first logarithm in (79) becomes large, too, and a minimum of function $\theta_{\text {v.r. }}\left(\nu^{(-)}\right)$develops, corresponding to onset of a rainbow scattering. As for the second logarithm in (79), which raises at $\nu^{(-)} \rightarrow 0$, it does not lead to formation of a dependence $\theta_{\text {v.r. }}\left(\nu^{(-)}\right)$minimum - on the contrary, it makes the dependence steeper, and in the area of its significance the differential cross-section is small (exponentially). So, for the differential cross-section description it basically suffices to consider only two regions: the region where the last term of (79) dominates, and the region where the last term and $\frac{1}{2} \ln \frac{1}{e\left(1-\nu^{(-)}\right)}$in (79) are competing. On the $\theta_{\text {v.r. }}$ axis the mentioned regions are adjacent, and conjointly they should give almost the full picture of the differential cross-section variation. For completeness, one may consider also a third, asymptotic region of the the differential cross-section tail (orbiting region), where the first and the second logarithms of (79) dominate.

a. Rainbow region. The value of $\nu^{(-)}$corresponding to the rainbow angle is to be determined from condition

$$
\left.\frac{\partial \theta_{\text {v.r. }}}{\partial \nu^{(-)}}\right|_{\nu^{(-)}=\nu_{0}^{(-)}}=0
$$

which in application to expression (79) gives

$$
\frac{\nu_{0}^{(-)}}{1-\nu_{0}^{(-) 2}}-\frac{1}{\nu_{0}^{(-)}}+\ln \left(1+\nu_{0}^{(-)}\right)=\ln \frac{R}{R_{c}} \gg 1 .
$$

The approximate solution of Eq. (83) is

$$
\nu_{0}^{(-)} \approx 1-\frac{1}{2 \ln \frac{e^{5 / 4} R}{2 R_{c}}}+\mathcal{O}\left(\frac{1}{\ln ^{3} \frac{e^{5 / 4 R}}{2 R_{c}}}\right) .
$$

In vicinity of the found point $\nu_{0}^{(-)}$we may expand func-

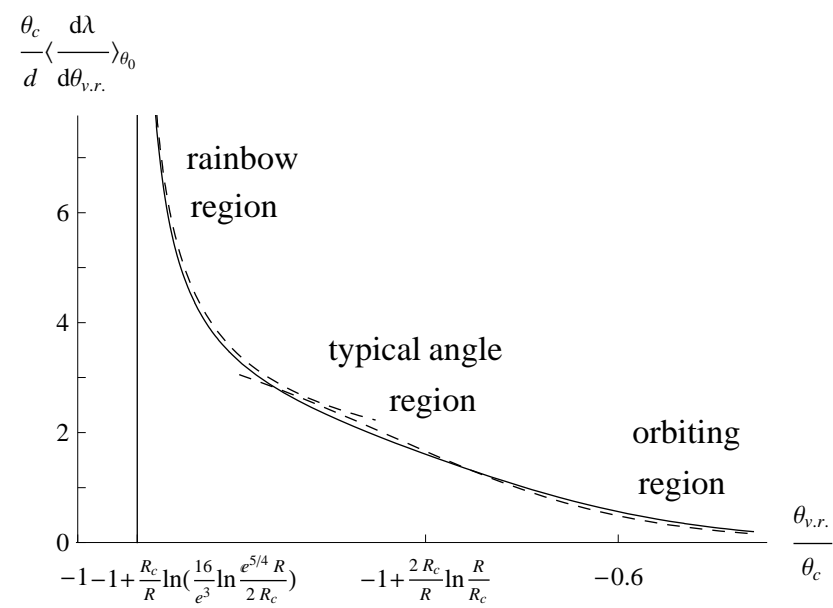

FIG. 10: The $\theta_{0}$-averaged differential cross-section of negatively charged particle scattering, at $R / R_{c}=25$ (solid curve). The axes scales are chosen so that areas under the curve is unity, as the total probability. The left-hand dashed curve is evaluated by the explicit approximate formula (86), the righthand dashed curve - by the explicit approximate formula (93). It is observed that those approximations actually overlap.

tion $\theta_{\text {v.r. }}\left(\nu^{(-)}\right)$up to a quadratic term

$$
\begin{aligned}
\left(\frac{\theta_{\text {v.r. }}}{\theta_{c}}+1\right) \frac{R}{2 R_{c}} & \approx \frac{1}{2} \ln \left(\frac{16}{e^{3}} \ln \frac{e^{5 / 4} R}{2 R_{c}}\right) \\
& +\left(\nu^{(-)}-\nu_{0}^{(-)}\right)^{2} \ln ^{2} \frac{e^{5 / 4} R}{2 R_{c}} \\
& +\mathcal{O}\left(\left(\nu^{(-)}-\nu_{0}^{(-)}\right)^{3} \ln ^{3} \frac{e^{5 / 4} R}{2 R_{c}}\right) .
\end{aligned}
$$

Now, expressing the pair of roots $\nu_{j}^{(-)}\left(\theta_{\text {v.r. }}\right)$ from the quadratic equation (85), one derives by formula (80) the behavior of the $\theta_{0}$-averaged differential cross-section in vicinity of the rainbow angle:

$$
\frac{\theta_{c}}{d}\left\langle\frac{d \lambda}{d \theta_{\text {v.r. }}}\right\rangle_{\theta_{0}} \approx \frac{\sqrt{R / 2 R_{c}}}{\ln \frac{e^{5 / 4 R}}{2 R_{c}} \sqrt{\frac{\theta_{\mathrm{v} . \mathrm{r}}}{\theta_{c}}+1-\frac{R_{c}}{R} \ln \left(\frac{16}{e^{3}} \ln \frac{e^{5 / 4 R}}{2 R_{c}}\right)}} .
$$

The domain of applicability of this approximation is determined from Eq. (85) by demanding the third-order term to be small compared with the second-order one:

$$
\left(\frac{\theta_{\text {v.r. }}}{\theta_{c}}+1\right) \frac{R}{R_{c}}-\ln \left(\frac{16}{e^{3}} \ln \frac{e^{5 / 4} R}{2 R_{c}}\right) \ll 1 \quad \text { (rainbow region). }
$$

Function (86) is shown by the left-hand dashed curve in Fig. 10,

b. Typical angle region. On the other hand, if $\nu^{(-)}$ is not too close to 1 , i. e. under condition

$$
\left(\frac{\theta_{\mathrm{v} . \mathrm{r} .}}{\theta_{c}}+1\right) \frac{R}{R_{c}}-\ln \left(\frac{16}{e^{3}} \ln \frac{e^{5 / 4} R}{2 R_{c}}\right) \gtrsim 1 \quad \text { (typical angles) }
$$


opposite to (87), all the terms in (79) except the second logarithm (singular at $\nu^{(-)} \rightarrow 0$ ) may reasonably be approximated by their Taylor expansions up to linear terms - say, about the unit interval midpoint $\nu_{\text {mid }}^{(-)}=\frac{1}{2}[30]$ :

$$
\theta_{\mathrm{v} . \mathrm{r} .} \approx-\theta_{c}\left[1-\frac{2 R_{c}}{R}\left(\ln \frac{\sqrt{3} R}{\nu^{(-)} R_{c}}-\frac{11}{6}-\nu^{(-)} \ln \frac{2 R}{3 e^{2 / 3} R_{c}}\right)\right] .
$$

Then, for determination of the inverse function $\nu^{(-)}\left(\theta_{\text {v.r. }}\right)$ one obtains the following simplified equation:

$$
\left(\frac{\theta_{\mathrm{v} . \mathrm{r} .}}{\theta_{c}}+1\right) \frac{R}{2 R_{c}}+\frac{11}{6} \approx \ln \frac{\sqrt{3} R}{\nu^{(-)} R_{c}}-\nu^{(-)} \ln \frac{2 R}{3 e^{2 / 3} R_{c}} .
$$

Here the r. h. s. is a monotonic function of $\nu^{(-)}$, so, in contrast to the exact equation (79), the simplified equation (90) has only one root (the second, lost root gives a small contribution to the differential crosssection). The solution to Eq. (90) expresses through the Lambert (product $\log$ ) function $W(s)$ defined as a solution to equation $\ln s=\ln W+W$, and incorporated in many computational software packages:

$\nu^{(-)} \approx \frac{1}{\ln \frac{2 R}{3 e^{2 / 3} R_{c}}} W\left(\frac{\sqrt{3} R \ln \frac{2 R}{3 e^{2 / 3} R_{c}}}{R_{c}} e^{-\frac{11}{6}-\frac{R}{2 R_{c}}\left(\frac{\theta_{\mathrm{v}, \mathrm{r}}}{\theta_{c}}+1\right)}\right)$.

(Mind that $\theta_{\text {v.r. }} / \theta_{c}$ is negative and close to -1 ). From the definition of $W$, its asymptotic behavior in different regions derives as

$$
W(s)= \begin{cases}s-\mathcal{O}\left(s^{2}\right) & s \ll 1 \\ \ln \frac{s}{\ln \frac{s}{\ln \frac{s}{\cdots}}} & s \gg 1\end{cases}
$$

and its derivative

$$
W^{\prime}(s)=\frac{1}{s(1+1 / W(s))} .
$$

Therefore, over the typical angle region the $\theta_{0}$-averaged differential cross-section is cast as

$$
\frac{\theta_{c}}{d}\left\langle\frac{d \lambda}{d \theta_{\text {v.r. }}}\right\rangle_{\theta_{0}}=\theta_{c}\left|\frac{d \nu^{(-)}}{d \theta_{\text {v.r. }}}\right| \approx \frac{R}{2 R_{c} \ln \frac{2 R}{3 e^{2 / 3} R_{c}}} \frac{1}{(1+1 / W)},
$$

the argument of $W$ being the same as in Eq. (91).

The full width of the differential cross-section as can be inferred from Eq. (93), and is obvious already from Eq. (79), amounts

$$
\triangle \theta_{\text {v.r. }} \sim \theta_{c} \frac{2 R_{c}}{R} \ln \frac{R}{R_{c}} \quad \text { (negatively charged particles), }
$$

which at $R / R_{c}>e^{\pi} \approx 23$ is larger than width (64) for positively charged particles.

The behavior of function (93) in region (88) is shown in Fig. 10 by the right-hand dashed curve. It describes the actual distribution quite accurately. In the orbiting region asymptotics, however, Eq. (93) errs by a factor of $e^{5 / 6} / \sqrt{3} \approx 1.3$ (see Eq. (96) below), so applicability of (93) is restricted by the bound

$$
\frac{R}{2 R_{c}}\left(\frac{\theta_{\text {v.r. }}}{\theta_{c}}+1\right) \lesssim \ln \frac{R}{R_{c}} \quad \text { (typical angles) }
$$

in addition to (88).

One may anticipate formation of a shoulder in the distribution (93) at sufficiently large $R / R_{c}$, since then the argument of $W$ can be large and $1+1 / W \rightarrow 1$, making the differential cross-section $\theta_{\text {v.r. }}$-independent. However, $W(s)$ reaches $5(\gg 1)$ only at $s \sim 700$, so a shoulder in $\left\langle d \lambda / d \theta_{\text {v.r. }}\right\rangle_{\theta_{0}}$ may form up only at $R / R_{c} \gtrsim 100$.

c. Orbiting region. Finally, asymptotically large $\theta_{\text {v.r. }}$ are generated in the regions $\nu^{(-)} \rightarrow 0$ and $\nu^{(-)} \rightarrow 1$, where there are logarithmically rising terms in the relation (79). Examine first the region $\nu^{(-)} \rightarrow 0$. In this limit one may let $\nu^{(-)}=0$ everywhere in (79) except in $\ln \frac{1}{\nu^{(-)}}$, and the reduced equation

$$
\theta_{\mathrm{v} . \mathrm{r} .} \simeq-\theta_{c}\left(1-\frac{2 R_{c}}{R} \ln \frac{R}{e R_{c} \nu^{(-)}}\right)
$$

is easily solved:

$$
\nu^{(-)} \simeq \frac{R}{e R_{c}} e^{-\frac{R}{2 R_{c}}\left(\frac{\theta_{\mathrm{v}, \mathrm{r} .}}{\theta_{c}}+1\right)} .
$$

Differentiating that expression with respect to $\theta_{\text {v.r. }}$, the differential cross-section asymptotics results as

$$
\frac{\theta_{c}}{d}\left\langle\frac{d \lambda}{d \theta_{\text {v.r. }}}\right\rangle_{\theta_{0}} \simeq \frac{R^{2}}{2 e R_{c}^{2}} e^{-\frac{R}{2 R_{c}}\left(\frac{\theta_{v} . \text { r. }}{\theta_{c}}+1\right)} \quad \text { (orbiting region) }
$$

holding if

$$
\frac{R}{2 R_{c}}\left(\frac{\theta_{\text {v.r. }}}{\theta_{c}}+1\right) \gg \ln \frac{R}{R_{c}} \quad \text { (orbiting region). }
$$

As for the contribution from region $\nu^{(-)} \rightarrow 1$, which can be treated in a completely analogous manner, it equals

$$
\frac{8 R}{e^{4} R_{c}} e^{-\frac{R}{R_{c}}\left(\frac{\theta_{\mathrm{v}} \cdot \mathrm{r} .}{\theta_{c}}+1\right)},
$$

and is definitely negligible compared to (96), so Eq. (96) is the complete asymptotic result.

\section{Comparison with experiment}

The only measurement of negatively charged particle volume reflection is [4]. It gave, for $\mathrm{Si}$ (110) orientation, $R / R_{c} \approx 70$, the volume reflection angle mean value $\left|\left\langle\theta_{\text {v.r. }}\right\rangle\right|=0.66 \theta_{c}$. This is significantly smaller than our expectation (and other simulations, as quoted in [15]) $\left|\left\langle\theta_{\mathrm{v} . \mathrm{r}}\right\rangle\right| \approx \theta_{c}$ (yet minus $\sim 8 \%$ correction for finite $R_{c} / R$, which does not matter anyway). Of course, if we treat $\left|F_{\text {max }}\right|$ as an adjustable parameter of the model, we might 
achieve agreement with the experiment, but a physical recipe for this is yet lacking.

As for numerical calculation results for the final beam profile, there is lack thereof for negatively charged particles at $R \gg R_{c}$ and free of multiple scattering (and with boundary conditions congruent with typical experimental ones) [31]. We hope to see such results in near future.

\section{HIGH-ENERGY PASSAGE LIMIT (PERTURBATIVE DEFLECTION)}

In conclusion, we will briefly comment on behavior of the function $\theta_{\text {v.r. }}(b)$ in the opposite, high-energy limit,

$$
\frac{d}{2 \delta}=\frac{R}{R_{c}} \ll 1 .
$$

Thereunder, the deflection becomes perturbative (and better viewed in Cartesian coordinates, without the reference to a centrifugal force notion), and for positive and negative particles it must be equal in magnitude but opposite in sign. That is confirmed by Figs. 4 and Fig. 11

The specific expression for the dependence $\theta_{\text {v.r. }}(b)$ in this limit was obtained in [16] (Eqs. (18-19)), in the Cartesian coordinate framework:

$$
\begin{aligned}
\theta_{\text {v.r. }}\left(\theta_{0}, b\right) & \rightarrow \theta_{\text {Born }}\left(\theta_{0}, b\right)=\frac{1}{E} \int_{-\infty}^{\infty} d z F(b, z) \\
& = \pm 4 \frac{\sqrt{2 R d}}{R_{c}} \zeta\left(-\frac{1}{2},\left\{\frac{1}{2}+\frac{R}{2 d} \theta_{0}^{2}+\frac{b}{d}\right\}_{\mathrm{f}}\right)
\end{aligned}
$$

(again, the braces indicate taking the fractional part). It involves the Hurwitz (generalized Riemann) zetafunction at a negative value of its first argument, which may be defined, e. g., as a contour integral [17]

$$
\zeta(\alpha, v)=\frac{\Gamma(1-\alpha)}{2 \pi i} \int_{-\infty}^{(0+)} \frac{s^{\alpha-1} e^{v s}}{1-e^{s}} d s
$$

along a Hankel path [32]. Function (98b) is shown in Fig. 11 by dotted line. Note the identity $\zeta\left(-\frac{1}{2}, 0\right)=$ $\zeta\left(-\frac{1}{2}, 1\right)\left(\equiv \zeta\left(-\frac{1}{2}\right)\right)$, whereby function (98b) is everywhere continuous, but its derivative breaks at point $\frac{b}{d}=\frac{1}{2}-\left\{\frac{R \theta_{0}^{2}}{2 d}\right\}_{\mathrm{f}}$.

The second argument of $\zeta$-function in $98 \mathrm{~b}$ allows for a physical interpretation [16]:

$$
b_{\text {refl }}=b+\frac{R}{2} \theta_{0}^{2}
$$

is the particle impact parameter at a depth where the particle straight trajectory becomes tangential to the bent atomic planes (actually, $t_{\text {reff }}$ ). Should one pass to $b_{\text {refl }}$ instead of $b$, the dependence on $\theta_{0}$ disappears completely:

$$
\theta_{\text {v.r. }}\left(\theta_{0}, b\right)=\theta_{\text {v.r. }}\left(b_{\text {reff }}\right) \text {. }
$$

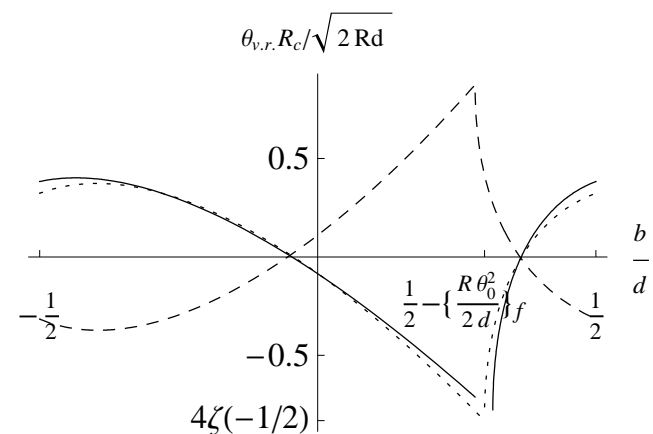

FIG. 11: Comparison of impact parameter dependencies of the reflection angle for positively charged (solid curve) and negatively charged (dashed curve) particles, at $R / R_{c}=1 / 20$. Dotted curve - approximation (98b), for positive particles. The functions displayed may be continuously periodically extended beyond the interval $\left(-\frac{1}{2}, \frac{1}{2}\right)$. The position of the fracture at $\frac{b}{d}=\frac{1}{2}-\left\{\frac{R \theta_{0}^{2}}{2 d}\right\}_{\mathrm{f}}$ corresponds to tangency of the particle near-straight trajectory to one of the bent atomic planes.

This contrasts with the case $R \gg R_{c}$ investigated in the previous section, where the $\theta_{0}$-dependence yet remained in a specific, casually located rainbow peak.

Here we will not contemplate demonstrating from expressions (36/50b) that the limit of $\theta_{\text {v.r. }}$ is indeed (98b). We only note that to this end one must implement significant cancellations between the pair of terms under the sum sign. One also notes that the second argument of $\zeta$-function in (98b) is just the limit of $\nu^{(+)}$:

$$
\lim _{\delta / d \rightarrow \infty} \nu^{(+)}=\left\{\frac{1}{2}+\frac{R}{2 d} \theta_{0}^{2}+\frac{b}{d}\right\}_{\mathrm{f}} .
$$

For negative particles the route to $98 \mathrm{~b}$ is a bit more intricate. In Fig. 11] we numerically compare approximation (98b) with the exact result - the agreement is quite convincing.

The comparison with the perturbative scattering pattern may also give an insight into the origin of the volume reflection phenomenon. The average of function (98) over the impact parameter $b$ turns out to be strictly zero (see 16]) - hence, in the high-energy limit signatures of the volume reflection completely disappear. That owes to the fact that $\int_{-d / 2}^{d / 2} d b F(b, z) \equiv 0$ at any given $z$ (regardless of the crystal bend). In contrast, at $R \gtrsim R_{c}$ the trajectory may not be viewed as merely straight [33], and the particle distribution is non-uniform over the crystal volume. In fact, "shadowed regions" not filled by the particles may appear at the inner side of bent potential ridges, in which the force acts in the positive direction. The deficit of a positively directed force on the beam then leads to the negative sign of the particle beam mean deflection angle. 


\section{EXPERIMENTAL OBJECTIVES AND PARAMETER OPTIMIZATION}

The alleged application of volume reflection is for highenergy particle beam extraction from accelerator beamlines, when that is not manageable locally with laboratory magnets (intra-crystal forces are much stronger). Thereat, the beam parameters (energy and angular divergence) are definite, while the crystal parameters have to be optimized in order to attain a suitable deflection quality.

Control of beam divergence accompanying the deflection. The mean deflection angle

$$
\left|\theta_{\text {v.r. }}\right| \sim \theta_{c}, \quad \theta_{c}=\underset{(\text { for } \operatorname{Si}(110))}{\sqrt{\frac{2 V_{0}}{E}} \approx 21 \mu \mathrm{rad} \sqrt{\frac{100 \mathrm{GeV}}{E}}}
$$

for a silicon crystal of orientation (110) depends only on the particle energy. The next parameter to care about is the angular spread acquired by the beam at the exit from the crystal. Neglecting the incoherent multiple scattering (to be estimated below), the spread $\triangle \theta_{\text {v.r. }}$, given by equations (64) and (94), for a given beam energy and crystal material depends only on the crystal bending radius. To derive a criterion for beam complete deflection, one may demand that the bulk of the dispersed beam (its both "edges") be deflected to the same side. That implies:

$$
R>4 R_{c} \quad \text { (for positively charged particles) }
$$

and

$$
R>2 R_{c} \ln \frac{R}{R_{c}} . \quad \text { (for negatively charged particles) }
$$

Relation (104) with the factor 4 was found previously by Maisheev [9] based on numerical simulation studies for protons. Our paper, thereby, offers a formal justification for that empirical relation, although within a framework of a simplified model. The emerging ratio

$$
\mathrm{q}=\frac{\max \left|\theta_{\text {v.r. }}\right|}{\triangle \theta_{\text {v.r. }}}= \begin{cases}\frac{R}{4 R_{c}} & \text { for pos. charged particles } \\ \frac{2 R_{c} \ln \frac{R}{R_{c}}}{} & \text { for neg. charged particles }\end{cases}
$$

quantifies the steering quality. But for the deflection to be neat, one should have a substantial q

$$
\mathrm{q} \gtrsim 3 \quad \text { (neat deflection), }
$$

entailing for the crystal curvature radius

$$
R \gtrsim 10 \div 15 R_{c} \text {. }
$$

In demonstrational experiments [3] the latter requirement was marginally satisfied.
Sufficient crystal thickness. The crystal thickness $(L)$, it to be kept as low as possible in order to minimize the multiple scattering. However, a lower bound for $L$ results from the requirement that the volume reflection has space to develop, i. e., the "thick crystal limit" holds, in the sense of Sec. IIIC] Thereto, the crystal bending half-angle $\frac{L}{2 R}$ needs be larger than the critical angle $\theta_{c}$, which is $L$-independent. Thus,

$$
L \gg 2 R \theta_{c}=\frac{R}{R_{c}} d \sqrt{\frac{E}{2 V_{0}}} \approx \frac{R}{4 R_{c}} 3.6 \mu \mathrm{m} \sqrt{\frac{E}{\mathrm{GeV}}}
$$

(thick crystal, volume reflection saturation).

If we regard here $\frac{R}{4 R_{c}}=\mathrm{q}$ as fixed, in general $L$ grows with the energy. At $E \sim 10^{2} \div 10^{3} \mathrm{GeV}$ (RHIC, Tevatron) the minimal thickness amounts only to $(0.036 \div 0.1 \mathrm{~mm}) \mathrm{q}$. Note that crystals as thin as $30 \mathrm{~mm}$ are manufacturable (as described in [18]). For $E \sim 10 \mathrm{TeV}(\mathrm{LHC})$ and $\mathrm{q} \sim 3$ minimal $L$ reaches the value of $1 \mathrm{~mm}$.

Reduction of multiple scattering. To quantify the impact of incoherent, random multiple scattering, we have to evaluate the characteristic ratio 34$]$

$$
\begin{aligned}
\frac{\theta_{x}^{\text {mult }}}{\theta_{c}} & \approx \frac{13.6 \mathrm{MeV}}{\sqrt{2} E} \sqrt{\frac{L}{93.6 \mathrm{~mm}}} \sqrt{\frac{E}{2 V_{0}}} \\
& \approx 0.47 \sqrt{\frac{100 \mathrm{GeV}}{E} \frac{L}{\mathrm{~mm}}} \quad(\mathrm{Si}(110)) .
\end{aligned}
$$

If the latter square root does not exceed unity (in experiments $[3]$ it is $\approx 1$ ), the multiple scattering does not spoil coherent beam deflection. We conclude that for $E \gtrsim 100 \mathrm{GeV}$ crystal lengths up to $2 \mathrm{~mm}$ are multiple scattering safe, i. e., $\theta_{x}^{\text {mult }} / \theta_{c}<1$.

Besides aggregate deflection, it would be interesting to experimentally investigate the intrinsic volume-reflected beam shape, and in particular to check the shape dependence on the particle charge sign (cf. Figs. 7 and 10). The main problem here is that at $\mathrm{q} \gg 1$ the final beam half divergence $\frac{1}{2} \triangle \theta_{\text {v.r. }}$ is $2 q$ times smaller than the mean deflection angle, and so is sooner overtaken by the multiple scattering, making the profile Gaussian and particle charge sign independent. For this not to happen, one needs condition

$$
\frac{\theta_{x}^{\text {mult }}}{\frac{1}{2} \triangle \theta_{\text {v.r. }}} \sim 2 \mathrm{q} \frac{\theta_{x}^{\text {mult }}}{\theta_{c}} \ll 1,
$$

with $\theta_{x}^{\text {mult }} / \triangle \theta_{\text {v.r. }}$ to be inferred from (110). Hence, for the present purpose we should not strive for large $\mathrm{q}$, granted that the final beam profile is not very sensitive to $\mathrm{q}$ at $\mathrm{q}>1$. So, $\mathrm{q} \simeq 1.2$ seem to be good enough. Equally well, in order to raise the angular resolution we should use moderate energies. Say, $E=50 \mathrm{GeV}$ is ultra-relativistic enough. To reduce multiple scattering, we can take a thin crystal with $L=30 \mu \mathrm{m}$, which marginally satisfies (109). This gives $\theta_{c} \approx 3 \cdot 10^{-5} \mathrm{rad}, \Delta \theta_{\text {v.r. }} \approx 2.5 \cdot 10^{-5} \mathrm{rad}$, and 
$\frac{\theta_{x}^{\text {mult }}}{\frac{1}{2} \Delta \theta_{\mathrm{v}, \mathrm{r} .}} \approx 0.3$ (small enough). But one has to control initial particle impact angles with an accuracy a few times better than $\triangle \theta_{v . r .}$. This may be difficult to achieve via initial beam collimation alone, so one may need to apply event selection procedures (cf. [4]).

\section{SUMMARY}

Based on the model of a purely parabolic continuous potential in a bent crystal, we have gained a lot of information about the volume reflection phenomenon, for cases of positively and negatively charged particles. First, we have obtained an explicit expression (26) for particle trajectories. From the solution for trajectory, in particular, we have derived the particle final deflection angle as a function of the particle impact parameter and energy, in form of sums (36, 50b). Asymptotic behavior of those sums at $R \gg R_{c}$ was explored, and asymptotic values for the volume reflection angle were found. They equal: $-\frac{\pi}{2} \theta_{c}$ for positive particles, and $-\theta_{c}$ for negative particles. This agrees within $\sim 20 \%$ with the existing results of numerical simulation using more realistic continuous potentials [2, 9] and with experiment for positive particles [3] (though there is an indication of worse agreement for negative particles [4]). $20 \%$ is about the same accuracy as for approximating the continuous potential by a parabola. Yet we have evaluated the next-to-leading order correction in parameter $R_{c} / R$, which depends on the impact parameter, and, by averaging over impact parameters, we determined asymptotic shape of the final beam. This in particular yields the mean volume reflection angle dependence on $R_{c} / R$, which appears to be linear - in general agreement with experiment 14] (see Fig. (8).

In course of investigation of the final beam shape, we have discovered various singularities in its profile, which moreover are particle charge dependent. First of all, we had to deal with the problem that, in principle, the final beam profile may contain a visible admixture of boundary dependent effects ("randomly" located peaks). However, we have proved the statement that boundary effects get completely erased in the differential cross-section averaged over a tiny interval of incident angles $\theta_{0}$ (condition (69)), or, analogously, due to a bit of multiple scattering before the volume reflection region. Therewith, the averaging over impact parameters becomes equivalent to averaging over parameters $\nu^{( \pm)}$(i. e., transverse energy), and we were able to analytically deduce the final beam profile for positive and for negative particles. For negatively charged particles it is asymmetric, exhibiting a spike on its outer edge, corresponding to the rainbow scattering, and an exponential tail on the inner side, corresponding to orbiting (Fig. 10). For positive particles, the final beam has a rectangular profile (Fig. 7). But in actual practice, with the account of continuous potential smearing in vicinity of the atomic planes, one expects appearance of a weak rainbow spike and orbiting tail for positive particles, as well.

Towards practical applications and further experimental investigations, we have made a few numerical estimates. They indicate that for usage of a bent crystal as a coherent beam deflector, one needs a relation between the main parameters

$$
\frac{L}{1 \mathrm{~mm}}<\frac{E}{100 \mathrm{GeV}}<\frac{R}{\mathrm{~m}},\left(\frac{20 \mu \mathrm{rad}}{\sigma_{0}}\right)^{2}
$$

( $\sigma_{0}$ is the r.m.s. angular deviation in the initial beam). The better those inequalities are met, the higher is the deflection quality. If one becomes interested in investigation of the final beam intrinsic shape, generated by the continuous potential alone, those inequality must be satisfied strongly, but minding existence of technical lower limits for $L$ and $\sigma_{0}$. This suggests an optimal energy about $50 \mathrm{GeV}$; experiments are to be carried out simultaneously with particles of both charge signs $\left(e^{ \pm}, \pi^{ \pm}\right)$.

There are many respects in which the model solution described herein can be improved. First of all, it is straightforward to add to the simple parabolic potential a second parabolic section - either to round off the potential in vicinity of atomic planes, or to describe the potential of Si crystal in (111) planar orientation, which is of practical importance, too. As a next step - at least a perturbative account of incoherent scattering processes is desirable. But at the same time, even in the present form, the theory (trajectories derived in Sec. III) seems suitable, e. g., for study of electromagnetic radiation emitted by a volume-reflected particle.

\section{Appendix A: Formal procedure of $\theta_{0}$-averaging}

In Sec. IVA we had obtained, for positively charged particles, the scattering differential cross-section in form

$$
\frac{d \lambda}{d \theta_{\text {v.r. }}}=\frac{R}{2 \pi \theta_{c} R_{c}} \sum_{m} \frac{1}{\left|d \nu^{(+)} / d b\right|_{b=b_{m}\left(\nu^{(+)}\left(\theta_{\text {v.r. }}\right), \theta_{0}\right)}} .
$$

Our objective now is to straightforwardly compute the sum involved hereat for the specific function $\nu^{(+)}\left(b, \theta_{0}\right)$ given by Eq. (35), first for an arbitrary $\theta_{0}$, and then average it over $\theta_{0}$, in order to justify our assertion that combined averaging over $b$ and $\theta_{0}$ (within tiny interval (69)) is equivalent to averaging over $\nu^{(+)}$.

To begin with, let us find the roots $b_{m}$ explicitly. Eq. (35) is equivalent to

$$
\frac{\tau^{2} \theta_{0}^{2}+(b+\delta)^{2}-\left(\frac{d}{2}-\delta\right)^{2}}{2 \delta d}=\nu^{(+)}+m
$$

with $m$ an integer, solution of which is straightforward:

$$
b_{m}\left(\nu^{(+)}, \theta_{0}\right)=-\delta \pm \sqrt{2 \delta d\left(\nu^{(+)}+m\right)+\left(\frac{d}{2}-\delta\right)^{2}-\tau^{2} \theta_{0}^{2}} .
$$


Here the sequence of $m$ begins with a smallest integer $m_{0}$ at which the radicand in (A3) is yet positive, viz.

$$
m_{0}=\left\lfloor\frac{\tau^{2} \theta_{0}^{2}-\left(\frac{d}{2}-\delta\right)^{2}}{2 \delta d}-\nu^{(+)}\right\rfloor+1 .
$$

The upper limit of $m$ in Eq. A3 equals to the largest integer at which yet $b<\frac{d}{2}$ (for a branch with the "+" sign in front of the root in Eq. (A3)), and $b>-\frac{d}{2}$ (for a branch with the "-" sign). That yields, correspondingly, values

$$
\begin{aligned}
& m_{\max 1}=\left\lfloor\frac{\tau^{2} \theta_{0}^{2}}{2 \delta d}-\nu^{(+)}\right\rfloor, \\
& m_{\max 2}=m_{\max 1}+1 .
\end{aligned}
$$

The number of terms in the sums from (A4) to A5, A6) is large:

$$
m_{\max 1,2}-m_{0}=\frac{d}{8 \delta}+\mathcal{O}(1) \equiv \frac{R}{4 R_{c}}+\mathcal{O}(1) \gg 1 .
$$

Next, values of derivative $\frac{d \nu^{(+)}}{d b}$ in points $b_{m}$ are easily evaluated, noticing that the fractional part operator in $\nu^{(+)}$is inconsequential for derivatives [35]. Differentiating (A2) gives

$$
\left.\frac{d \nu^{(+)}}{d b}\right|_{b=b_{m}}=\frac{b_{m}+\delta}{\delta d}
$$

Identity

$$
\frac{1}{\left|d \nu^{(+)} / d b\right|_{b=b_{m}}}=\left|\frac{\partial b}{\partial m}\right|
$$

suggests that upon substitution to (A1) one may expect

$$
\begin{aligned}
\left(\sum_{m=m_{0}}^{m_{\max 1}}+\sum_{m=m_{0}}^{m_{\max 2}}\right) \frac{1}{\left|d \nu^{(+)} / d b\right|_{b=b_{m}}} & \approx 2 \sum_{m=m_{0}}^{m_{\max 1}} \frac{\partial b}{\partial m} \\
& \approx 2 \int_{0}^{d / 2} d b=d,
\end{aligned}
$$

as we anticipated in Sec. IVA, but to accommodate the dependence on $\theta_{0}$, we need to carry out the calculation more precisely.

Through (A3, A1), the differential cross-section assumes the form

$$
\begin{aligned}
& \frac{d \lambda\left(\theta_{\text {v.r. }}, \theta_{0}\right)}{d \theta_{\text {v.r. }}}=\frac{R}{2 \pi \theta_{c} R_{c}} \\
& \cdot\left(\sum_{m=0}^{m_{\max 1}}+\sum_{m=0}^{m_{\max 2}}\right) \frac{\delta d}{\sqrt{2 \delta d\left(\nu^{(+)}+m\right)+\left(\frac{d}{2}-\delta\right)^{2}-\tau^{2} \theta_{0}^{2}}} \\
& \approx \frac{d}{2 \pi \theta_{c}} \sqrt{\frac{R}{R_{c}}} \sum_{m=0}^{R / 4 R_{c}} \frac{1}{\sqrt{m+\alpha\left(\nu^{(+)}\left(\theta_{\text {v.r. }}\right), \theta_{0}\right)}} \quad(\mathrm{A} 10) \\
& \text { with } \\
& \alpha\left(\nu^{(+)}\left(\theta_{\text {v.r. }}\right), \theta_{0}\right)=1-\left\{\frac{\tau^{2} \theta_{0}^{2}-\left(\frac{d}{2}-\delta\right)^{2}}{2 \delta d}-\nu^{(+)}\right\}_{\mathrm{f}} \\
& \equiv\left\{\frac{\left(b \left(\nu^{(+)}\left(\theta_{\text {v.r. } \left.\left.), \theta_{0}\right)+\delta\right)^{2}}^{2 \delta d}\right\}_{\mathrm{f}}(\mathrm{A} 11)\right.\right.}{}\right.
\end{aligned}
$$

Outside the interval

$$
-\frac{\pi}{2} \theta_{c}<\theta_{\text {v.r. }}<-\frac{\pi}{2} \theta_{c}\left(1-\frac{4 R_{c}}{R}\right)
$$

there are no roots to equation $\nu^{(+)}\left(\theta_{\text {v.r. }}\right)$, so the differential cross-section vanishes as an empty sum.

At a large upper limit the sum in (A10) grows as $\sqrt{R / R_{c}}$, whereas the difference

$$
\sum_{m=0}^{R / 4 R_{c}} \frac{1}{\sqrt{m+\alpha}}-\sqrt{\frac{R}{R_{c}}} \underset{R / R_{c} \rightarrow \infty}{\longrightarrow} \zeta\left(\frac{1}{2}, \alpha\right)
$$

tends to a finite limit (it may be categorized as the Hurwitz, or generalized Riemann, zeta-function, see also definition (99)). Thereby, we may cast (A10) as

$$
\frac{d \lambda\left(\theta_{\text {v.r. }}, \theta_{0}\right)}{d \theta_{\text {v.r. }}} \approx \frac{d}{2 \pi \theta_{c}} \frac{R}{R_{c}}\left(1+\sqrt{\frac{R_{c}}{R}} \zeta\left[\frac{1}{2}, \alpha\left(\theta_{\text {v.r. }}, \theta_{0}\right)\right]\right)
$$

Here $\sqrt{R_{c} / R} \ll 1$, and the unity in parentheses (A14) corresponds to the result anticipated in (A9). But one should take care that at $\alpha \rightarrow 0$ function $\zeta\left(\frac{1}{2}, \alpha\right)$ blows up as $\frac{1}{\sqrt{\alpha}}$. So, the correction in the parentheses in (A14) can not be regarded as everywhere small.

Now, we turn to the issue that $\alpha$ is $\theta_{0}$-dependent. When $\theta_{0}$ varies (at $\theta_{\text {v.r. }}$ fixed) even in a narrow interval (see (67)), $\alpha$ uniformly and repeatedly scans its definition interval from 0 to 1 . Hence, the averaging over $\theta_{0}$ is equivalent to the integration over $\alpha$ from 0 to 1 . By virtue of the property

$$
\int_{0}^{1} d \alpha \zeta\left(\frac{1}{2}, \alpha\right) \equiv 0
$$

checkable from definition (A13), we have

$$
\begin{aligned}
& \left\langle\frac{d \lambda}{d \theta_{\text {v.r. }}}\right\rangle_{\theta_{0}}=\int_{0}^{1} d \alpha \frac{d \lambda}{d \theta_{\text {v.r. }}} \\
& =\frac{R d}{2 \pi \theta_{c} R_{c}} \Theta\left(\theta_{\text {v.r. }}+\frac{\pi}{2} \theta_{c}\right) \Theta\left(-\theta_{\text {v.r. }}-\frac{\pi}{2} \theta_{c}\left(1-\frac{4 R_{c}}{R}\right)\right) .
\end{aligned}
$$

Thereby, we arrive at equation (71), without any corrections $\sim \sqrt{R_{c} / R}$, q.e.d.

For negatively charged particles there arises a sum similar to that of (A1), though the dependence $\nu^{(-)}(b)$ differs a bit from $\nu^{(+)}(b)$. Nonetheless, the averaging procedure is completely analogous, the non-averaged differential cross-section equals (81) times the parentheses factor of (A14). Upon the $\theta_{0}$-averaging, through (A14) again, we arrive at Eq. (81). 
[1] E. N. Tsyganov, Fermilab Report No. TM-682, 1976 (unpublished); Fermilab Report No. TM-684, 1976 (unpublished); V. M. Biryukov, Yu. A. Chesnokov, V. I. Kotov. Crystal Channeling and its Application at HighEnergy Accelerators. Springer, Berlin, 1996; X. Artru, S. P. Fomin, N. F. Shul'ga, K. A. Ispirian, and N. K. Zhevago, Phys. Rep. 412 (2005) 89.

[2] A. M. Taratin and S. A. Vorobiev. Nucl. Instrum. Methods B 26 (1987) 512.

[3] Yu. M. Ivanov et al. Phys. Rev. Lett. 97 (2006) 144801; W. Scandale et al. Phys. Rev. Lett. 98 (2007) 154801.

[4] W. Scandale et al. Phys. Lett. B 681 (2009) 233.

[5] A. M. Taratin and W. Scandale. Nucl. Instrum. Methods B 262 (2007) 340.

[6] M. B. H. Breese and V. M. Biryukov. Nucl. Instrum. Methods B 263 (2007) 395; 265 (2007) 485.

[7] W. Scandale et al. Phys. Rev. Lett. 102 (2009) 084801; W. Scandale and M. Prest, CERN Report No. CERNSPSC-2008-014/SPSC-P-335, 2008.

[8] J. Lindhard, Kongel. Dan. Vidensk. Selsk., Mat.-Fys. Medd. 34(14) (1965) 1-64.

[9] V. A. Maisheev. Phys. Rev. ST Accel. Beams 10 (2007) 084701.

[10] A. M. Taratin. Phys. Part. Nucl. 29 (1998) 437; E. Bagli, V. Guidi, and V. A. Maisheev. Phys. Rev. E 81 (2010) 026708 .

[11] V. A. Maisheev. arXiv:physics/0607009v1.

[12] K. W. Ford and J. A. Wheeler. Ann. Phys. 7 (1959) 259; N. F. Mott, H. S. W. Massey. The Theory of Atomic Collisions. Clarendon, Oxford, 1965; R. G. Newton. Scattering Theory of Waves and Particles. Springer, N. Y., 1982; H. M. Nussenzveig. Diffraction Effects in Semi-Classical Scattering. Univ. Press, Cambridge, 1992.

[13] G. V. Kovalev. arXiv:physics.acc-ph/0712.0858; JETP Lett. 87 (2008) 87.

[14] W. Scandale et al. Phys. Rev. Lett. 101 (2008) 234801.

[15] W. Scandale et al. Phys. Rev. A 79 (2009) 012903.

[16] M. V. Bondarenco, Phys. Rev. A 81 (2010) 052903.

[17] F. W. J. Olver. Asymptotics and Special Functions. Academic Press, New York, 1974.

[18] Yu. M. Ivanov et al. JETP Lett. 84 (2006) 372; V. Guidi, A. Mazzolari, D. De Salvador, and A. Carnera, J. Phys. D 42 (2009) 182005.

[19] C. Amsler et al. [Particle Data Group], Phys. Lett. B 667 (2008) 1.

[20] The crystal has to be thin in order to avoid the strong influence of multiple scattering, but still it may be thick enough for the volume reflection to occur within the crystal volume and be independent of the boundaries. We shall quantify this requirement later on.

[21] We can turn to the issue of the initial beam divergence after we derive the scattering differential cross-section.

[22] At the very entrance to the crystal the force $z$-component may become comparable to $x$-component, in order to preserve the force nonvorticity but edge effects may certainly be neglected for a deeply penetrating particle.

[23] The condition thereof is that the Thomas-Fermi radius of the material atom be commensurable with the interatomic distance half width. In silicon that is the case we have. As for crystals of heavier elements such as tungsten, thereat the atomic radius is appreciably smaller, and the parabolic approximation for the inter-planar continuous potential is poor.

[24] Standardly [8], for a high-energy particle interacting with an oriented crystal, the particle wavelength shortness on the atomic scale makes the particle dynamics essentially classical, but yet non-perturbative, given the small angle of particle motion relative to a crystallographic direction and thus coherent action of atomic forces over long distances. Still, quantum effects may be viable in a special case when the particle transverse energy is very close to the height of an atomic potential barrier (the author is indebted to A.V. Shchagin for pointing this out in private conversation), but in any case, the classical calculation has to pave the way.

[25] Equation (1) in itself may apply to a non-ultra-relativistic motion, too, provided $E$ includes the particle rest energy (so, in the non-relativistic limit $E \rightarrow m$ ). But physically, in order not to complicate the analysis, we confine ourselves to (the most important) ultra-relativistic case in the present paper.

[26] Strictly speaking, the trajectory will not be exactly symmetric with respect to $t_{\text {refl }}$ because the distances from $t_{\text {refl }}$ to the crystal boundaries are in general different. However, contributions to $\theta_{\text {refl }}$ from crystal regions away from $t_{\text {refl }}$ are supposed to decrease sufficiently rapidly, and one expects existence of a "thick crystal limit" of $\theta_{\text {refl }}$, relevant in actual practice - see Sec. IV. In this paper, we content ourselves only to the "volume" contribution (33) to $\theta_{\text {refl }}$ and do not study any boundary effects. As we shall see later (Sec. IV] and Appendix), however, the omission of boundary effects requires certain care.

[27] The verbal description of the given effect for negative particle case is contained in [2] (end of Sec. 3), and a graphical illustration thereof appears in [9] (Fig. 5, trajectory 2 ). But relation with the general concept of orbiting (spiral scattering), as formulated in [12], was only noted in [13]. We don't go into discussion of possible experimental significance of orbiting here.

[28] Equation (55a) agrees with our definition of the $\theta$ angle and differs in sign from the conventionally defined angle in a centrally-symmetric field.

[29] In this subsection the sign alternative in $\frac{d}{2} \pm \delta$ corresponds to dealing with the first or with the second of the sums in $36 \mathrm{~b}$ ).

[30] More elegant expressions, at the same time accommodating for a correct asymptotics in the orbiting region, result when the Taylor expansion is carried out about the point $\nu^{(-)}=0$, but unfortunately, that approximation would be definitely less accurate at typical $\theta_{\text {v.r. }}$.

[31] Paper [9] does not exhibit negative particle results for $R>R_{c}$. In the pioneering work [2] the boundary conditions of are different from ours and from typical experimental arrangements: the authors consider particles entering the crystal face parallel to the bent atomic planes (a "half" volume reflection). That should produce a different final beam shape (with no rainbow singularity and more particles in the "orbiting" tail), but nonetheless, Fig. 4b of [2] is visually rather similar to our Fig. 10.

[32] Conventionally, the Hankel path is defined to begin in the complex $s$-plane at $-\infty(\arg s=-\pi)$, encircle the origin in the positive direction and return to $-\infty(\arg s=+\pi)$. 
[33] In the literature, sometimes, one meets an interpretation of the volume reflection phenomenon right in terms of a straight trajectory tangential to the bent crystalline planes in some point. Such an interpretation, although satisfactory for a symmetric installation of crystals with a large bending angle $\left(\sim 2 \theta_{0} \gg \theta_{c}\right)$, may be misleading for understanding of the underlying particle dynamics.

[34] For the (rms, plane) multiple scattering angle upon the particle over-barrier passage we crudely apply a formula for the scattering angle in an amorphous target made of the same material (silicon): $\theta_{x}^{\text {mult }}=\frac{13.6 \mathrm{MeV}}{\sqrt{2} E} \sqrt{\frac{L}{93.6 \mathrm{~mm}}}$ (as quoted in [19]).

[35] Strictly speaking, differentiation of finite discontinuities will give $\delta$-functional terms, but they will be imperceptible when inserted to the denominator of equation (65). 